\title{
Rate and state dependent friction and the stability of sliding between elastically deformable solids
}

\author{
James R. Rice ${ }^{a, b, *}$ Nadia Lapusta ${ }^{a}, K$. Ranjith ${ }^{\mathrm{a}}$ \\ ${ }^{a}$ Division of Engineering and Applied Sciences, Harvard University, Cambridge, MA 02138, USA \\ ${ }^{\mathrm{b}}$ Department of Earth and Planetary Sciences, Harvard University, Cambridge, MA 02138, USA
}

\begin{abstract}
We study the stability of steady sliding between elastically deformable continua using rate and state dependent friction laws. That is done for both elastically identical and elastically dissimilar solids. The focus is on linearized response to perturbations of steady-state sliding, and on studying how the positive direct effect (instantaneous increase or decrease of shear strength in response to a respective instantaneous increase or decrease of slip rate) of those laws allows the existence of a quasi-static range of response to perturbations at sufficiently low slip rate. We discuss the physical basis of rate and state laws, including the likely basis for the direct effect in thermally activated processes allowing creep slippage at asperity contacts, and estimate activation parameters for quartzite and granite. Also, a class of rate and state laws suitable for variable normal stress is presented. As part of the work, we show that compromises from the rate and state framework for describing velocity-weakening friction lead to paradoxical results, like supersonic propagation of slip perturbations, or to ill-posedness, when applied to sliding between elastically deformable solids. The case of sliding between elastically dissimilar solids has the inherently destabilizing feature that spatially inhomogeneous slip leads to an alteration of normal stress, hence of frictional resistance. We show that the rate and state friction laws nevertheless lead to stability of response to sufficiently short wavelength perturbations, at very slow slip rates. Further, for slow sliding between dissimilar solids, we show that there is a critical amplitude of velocity-strengthening above which there is stability to perturbations of all wavelengths. (C) 2001 Elsevier Science Ltd. All rights reserved.
\end{abstract}

Keywords: Tribology; B. Contact mechanics; B. Friction; B. Geological materials; C. Stability and bifurcation

\footnotetext{
* Corresponding author. Fax: +1-617-495-9837.

E-mail address: rice@esag.deas.harvard.edu (J.R. Rice).
} 


\section{Introduction}

This study considers two elastic solids, treated as half-space continua, in steady frictional sliding (Fig. 1) and studies their stability to linearized perturbation. This is done in the framework of rate and state dependent friction. It is argued that the rate and state framework is the physically relevant description of friction, particularly if quasi-static frictional responses as observed experimentally are to emerge as predicted responses on surfaces which undergo sufficiently slow slip rates. The existence of such quasi-static response is shown to be critically dependent on the positiveness of the "direct effect" (see below) in rate and state laws. Thus the physical basis of that effect is reviewed, in terms of thermally activated creep slippage at asperity contacts, and estimates of activation energy and volume are made for granite and quartzite.

The rate and state framework models the variations of frictional shear strength due to its dependence on slip rate and on the evolving properties of the contact population (Dieterich, 1979, 1981; Ruina, 1983). Dependence on those contact properties is represented as a dependence on a state variable, or variables (Ruina, 1983; Rice, 1983; Rice and Ruina, 1983; Tullis and Weeks, 1986; Heslot et al., 1994; Marone, 1998). A formulation of friction due to Oden and Martins (1985) can likewise be considered as a special limit of rate and state friction, provided that the small normal motion between sliding surfaces, included in that formulation, is simply considered as a state variable rather than also as a source of alteration of normal stress. Similar normal motion concepts are adopted in rate and state friction, in considering the dependence of strength on the dilatancy of a thin gouge of wear particles (Sleep, 1995; Segall and Rice, 1995). The rate and state effects can be thought of as, usually, small alterations of the friction strength from what would be predicted based on a constant friction

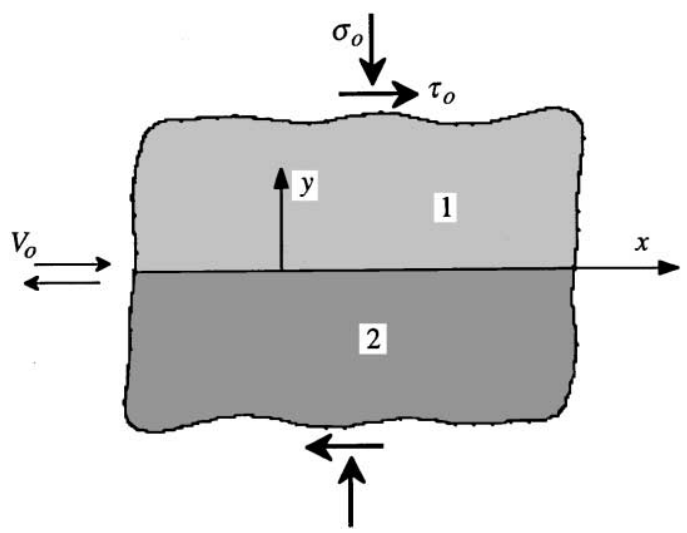

Fig. 1. Steady frictional sliding of two elastic half-spaces. Identical materials " 1 " and "2" are considered in the earlier parts of the paper, and dissimilar materials in the later parts. Orientation shown here is for analysis of in-plane $\exp (\mathrm{i} k x)$ perturbations of steady sliding at rate $V_{0}$ with uniform shear $\left(\tau_{0}\right)$ and normal $\left(\sigma_{0}\right)$ stresses. For analysis of anti-plane $\exp (\mathrm{i} k x)$ perturbations, the directions of $V_{0}$ and $\tau_{0}$ are taken perpendicular to the plane of the diagram. 
coefficient. In fact, those effects describe the actually gradual transition between what is often loosely called "static" and "kinetic" friction. Here, for some of our analyses, we want to use rate and state concepts to describe memory effects in the response not only to variations in slip rate, but also to variations in normal stress, as studied in experiments by Linker and Dieterich (1992), Prakash and Clifton (1992), Prakash (1998), Richardson and Marone (1999), and Bureau et al. (2000).

Two different kinds of studies are then presented using that class of friction laws. First, we address, by elastodynamic analysis, the problem of sliding between identical solids on an interface which exhibits velocity-weakening friction. The focus is on showing that the solution for the response to perturbation, when carried out using rate and state friction, converges to a quasi-static solution at sufficiently low slip rates. For such a quasi-static limit to exist, it is essential that friction exhibits positive direct velocity effect, that is, an abrupt change in sliding velocity should cause an abrupt change of the same sign in the friction strength. Such a property of friction is well-established experimentally. That is, for rapid enough changes in $V$ so as for the surfaces to be at the same population of asperity contacts (i.e., at a constant value of the state variable), the variation of friction strength $\tau$ with slip rate $V$ is generally found to involve a positive proportionality to $\ln (V)$. This dependence is plausibly attributed to the presence of a thermally activated creep process at stressed asperity contacts (Stesky, 1977; Heslot et al., 1994; Chester, 1994; Bréchet and Estrin, 1994; Baumberger, 1997; Sleep, 1997; Persson, 1998; Baumberger et al., 1999; Lapusta et al., 2000; Nakatani, 2001) as discussed further. We also demonstrate how compromises from the full rate and state constitutive framework, in the direction of classical friction laws like pure velocity-dependent friction, of velocity-weakening type, do not allow a quasi-static range and, in fact, lead to paradoxical prediction of supersonic propagation of slip pulses (as recognized already by Weertman, 1969, and Knopoff and Landoni, 1998), or to ill-posedness, depending on the strength of the velocity weakening.

As shown for anti-plane elastodynamics with rate and state friction by Rice and Ruina (1983), the loss of stability on velocity-weakening surfaces, as one considers the response to perturbations of progressively longer wavelengths, occurs as a Hopf bifurcation. At that bifurcation wavelength, the responses to perturbations propagate at a speed $c$ along the interface. We show that $c<c_{\mathrm{S}}$ (shear wave speed) for anti-plane perturbations, and $c<c_{\mathrm{R}}$ (Rayleigh surface wave speed) for in-plane perturbations. The fact that the shortest unstable wavelengths propagate at speeds which are, respectively, sub-shear or sub-Rayleigh suggests that fronts of slip perturbations might likewise do so, although this is strictly a conclusion reached here only in the context of linearized perturbation of a state of steady sliding.

The second kind of study involves slow frictional sliding along an interface between elastically dissimilar half-spaces. The dissimilar material interface is especially susceptible to frictional instability because spatially inhomogeneous slip causes a variation of normal stress along the interface (Comninou, 1977a, b; Comninou and Schmeuser, 1979; Weertman, 1980). Where normal stress is reduced, frictional slip is eased and the possibility for an instability is evident. The same effect does not exist for the sliding of identical elastic solids, as in the first kind of study here. Thus, for dissimilar materials in slow frictional sliding, we wish to examine stability versus instability due to both 
effects, the velocity dependence of friction and the coupling between inhomogeneous slip and alteration of normal stress.

The problem of stability of steady sliding between dissimilar materials has received much recent attention in theoretical modeling (Renardy, 1992; Martins et al., 1992, 1995; Adams, 1995; Martins and Simões, 1995; Simões and Martins, 1998; Cochard and Rice, 2000; Ranjith and Rice, 2001). That work has, mostly, neglected rate and state effects, instead assuming a constant coefficient of friction $f$, and has focused on the coupling between inhomogeneous slip and alteration of normal stress. Such a classical friction model, as we emphasize here, has no quasi-static range and must be analyzed in the framework of elastodynamics. The results for that classical model are that sliding is unstable to $\exp (\mathrm{i} k x)$ spatial perturbations $(x$ is the coordinate along the interface, in the sliding direction) for a very wide range of $f$ and of ratios of elastic constants. In fact, the time dependence of the response of slip rate or normal stress to perturbation is then of the form $\exp (p t)$, where $p=\hat{a}|k|-\mathrm{i} c$ with $\hat{a}$ and $c$ real and independent of $k$ (but dependent on $f$ and ratios of moduli). The widely occurring unstable situations correspond to $\hat{a}>0$. For those, the problem of response to a generic perturbation is ill-posed (Renardy, 1992; Martins and Simões, 1995; Simões and Martins, 1998; Cochard and Rice, 2000; Ranjith and Rice, 2001); it has no solution for any positive time if the Fourier strength of the perturbation falls off less rapidly than a negative exponential in $|k|$ at large $|k|$.

That result has already put a focus on how the Coulomb friction law should be modified to regularize such problems (Simões and Martins, 1998; Ranjith and Rice, 2001). Andrews and Ben-Zion (1997) reported numerical simulations of highly unstable slip pulses along interfaces between dissimilar materials that were shear-loaded below the friction threshold. Cochard and Rice (2000) showed that the model which Andrews and Ben-Zion used, with constant friction coefficient, would not give numerical convergence with grid refinement, due to the ill-posedness discussed. Cochard and Rice (2000) also showed that a reformulation of the problem using the regularized friction law of Ranjith and Rice (2001) did lead to convergent solutions with properties somewhat like those suggested by Andrews and Ben-Zion (1997). Extensive further studies along those lines, using the same regularized friction law, are reported by Ben-Zion (2001).

Thus for the sliding of dissimilar materials, it is appropriate to focus, as we do in this paper, on the combined, possibly destabilizing, effects of rate and state friction and coupling of normal stress to slip. That should be done in the context of elastodynamics, letting the quasi-static range emerge as a limit case. Here, in the interest of taking a simple first step, that problem is directly addressed as a quasi-static problem. Such a quasi-static limit should exist at sufficiently slow slip rates, as a consequence of the positive direct velocity effect in such laws. As mentioned above, the direct effect is generally found to involve a positive proportionality to $\ln (V)$. Thus $\partial \tau / \partial V$ (the derivative being taken at fixed normal stress $\sigma$ and state) has, at least approximately, the form $a \sigma / V$ at low enough (but, obviously, not too near zero) $V$. For rock friction, $a$ is typically of the order 0.01 at room temperature. In contrast, we may expect elastodynamic alterations of stress due to changes of slip rate to scale with $\mu / 2 c_{\mathrm{s}}$ where $\mu$ and $c_{\mathrm{s}}$ are representative shear modulus and shear wave speed, respectively. 
Thus it may be anticipated that the dynamic term will be dominated by $a \sigma / V$ when slip rates are slow enough to satisfy $V \ll 2 a \sigma c_{\mathrm{s}} / \mu$. For example, in the case of crustal rocks such as granite $\left(\mu=30 \mathrm{GPa}, c_{\mathrm{s}}=3 \mathrm{~km} / \mathrm{s}\right), 2 a \sigma c_{\mathrm{s}} / \mu \approx 2 \mathrm{~mm} / \mathrm{s} \times(\sigma / \mathrm{MPa})$. The slip rates should be much slower than that to be considered quasi-static, a stringent requirement at low $\sigma$.

In the quasi-static analysis (Section 4), we find that the sliding of dissimilar materials is always stable to the perturbation of the highest wavenumbers. As in the identical materials case, the loss of stability on velocity-weakening surfaces occurs at a critical wavenumber (as a Hopf bifurcation), and the sliding is unstable to perturbations of lower wavenumbers. However, for velocity-strengthening surfaces (for which the sliding of identical materials is stable to perturbations of all wavenumbers), the dissimilar materials case differs; for sufficiently low values of velocity strengthening, the sliding is still unstable to perturbations of low enough wavenumbers. This is the manifestation of more unstable behavior of the dissimilar materials case, where the reductions in normal stress contribute to frictional instability.

In Section 5, we adopt the elastodynamic formulation for dissimilar materials of Ranjith and Rice (2001) and confirm the result of the quasi-static analysis that the highest wave numbers are always stable provided that the sliding velocities are sufficiently low. This, in addition to the qualitative consideration above, gives further confidence in the appropriateness of the quasi-static analysis for sufficiently slow velocities. Furthermore, it means that the positive direct velocity effect of friction regularizes the elastodynamic description of sliding for dissimilar materials, at least for the sufficiently slow loading velocities.

\section{Rate and state dependent friction laws including variable normal stress, their linearization, and physical and empirical basis}

In this section a broad class of frictional constitutive laws is considered, all of which have the same linearized structure when applied to address small perturbations from steady sliding.

\subsection{General rate and state framework, including variable normal stress}

We are concerned with the relation between the shear stress $\tau$, compressive normal stress $\sigma$, and slip rate $V$ (in the same sense as $\tau$ ), and consider a range in which $\sigma>0$. We assume that the state of the asperity contacts can be characterized suitably by a single state variable $\psi$, such that

$$
\tau=F(\sigma, V, \psi)
$$

The presence of $\psi$ recognizes that strength is not uniquely determined by the two other variables, as shown in a host of experiments. The representation of laws for evolution of that state variable (or for a set of state variables, in more precise fits to experiments) has been much discussed in the literature. In the most common one-state-variable case, 
state evolution laws of the type

$$
\dot{\psi}=-G_{1}(\sigma, V, \psi)
$$

are used for sliding at constant $\sigma$.

We assume that $F$ has positive partial derivatives with respect to all three of its variables. $\partial F / \partial \sigma>0$ is agreeable enough, and $\partial F / \partial V>0$ is a firmly established experimental result (Dieterich, 1979; Ruina, 1983; Tullis and Weeks, 1986; Heslot et al., 1994; Marone, 1998), reflecting the positive instantaneous velocity dependence of processes at asperity contacts. $\partial F / \partial \psi>0$ merely sets a convention for the state variable; larger values mean greater strength, which is consistent with the usual interpretation of $\psi$ as a measure of the average maturity of the asperity contact population (more mature contacts are stronger).

For $G_{1}$ the only general assumption is that when $V$ and $\sigma$ are constant, the equation $\dot{\psi}=-G_{1}(\sigma, V, \psi)$ has a stable solution which evolves monotonically towards a definite value of $\psi$ (at which $\left.\dot{\psi}=-G_{1}=0\right)$. We call that the steady-state value $\psi_{\mathrm{ss}}=\psi_{\mathrm{ss}}(\sigma, V)$, satisfying $G_{1}\left(\sigma, V, \psi_{\mathrm{ss}}\right)=0$, and observe that for evolution of $\psi$ towards $\psi_{\mathrm{ss}}$, when $V$ and $\sigma$ are maintained constant, we must require $\partial G_{1} / \partial \psi>0$. For sliding in steady state at constant $V$ and $\sigma$, the frictional shear strength is thus

$$
\tau=F\left(\sigma, V, \psi_{\mathrm{ss}}(\sigma, V)\right) \equiv \tau_{\mathrm{ss}}(\sigma, V) .
$$

The same formulation is sometimes used also for sliding at variable $\sigma$, which is an essential feature of sliding between elastically dissimilar materials. However, experiments with variable normal stress histories (Linker and Dieterich, 1992; Prakash and Clifton, 1992; Prakash, 1998; Richardson and Marone, 1999; Bureau et al., 2000) show that there is also a memory dependence of variations in $\sigma$, and these effects cannot be captured by the above formulation.

Here we build on the suggestion by Linker and Dieterich (1992), based on their experiments with steps in normal stress on a sliding surface, of a way for accounting for such effects, while keeping the simplicity of a single state variable law. One continues to write $\tau=F(\sigma, V, \psi)$ as above but now assumes that the evolution of the state has the form (generalized from Linker and Dieterich, 1992)

$$
\dot{\psi}=-G(\sigma, \dot{\sigma}, V, \psi)=-G_{1}(\sigma, V, \psi)-\dot{\sigma} G_{2}(\sigma, V, \psi),
$$

where $G_{2}>0$. This allows for normal stress variations to alter the state (some functional forms, motivated by experiments, will be cited below). In sustained sliding at constant $V$ and $\sigma$, we have the same condition for a steady-state value $\psi_{\mathrm{ss}}=\psi_{\mathrm{ss}}(\sigma, V)$ as above, since that will satisfy $G\left(\sigma, 0, V, \psi_{\mathrm{ss}}\right)=G_{1}\left(\sigma, V, \psi_{\mathrm{ss}}\right)=0$, and hence, of course, have the same expression for $\tau_{\mathrm{ss}}$. The effect of adding to $G$ the term that is linear in $\dot{\sigma}$ is that there is a representation of the effect of very rapid (effectively instantaneous) changes in $\sigma$ which is given by integrating

$$
\mathrm{d} \psi / \mathrm{d} \sigma=-G_{2}(\sigma, V, \psi)
$$

at fixed $V$, or with a $V$ which changes instantaneously in some prescribed way with the change in $\sigma$. This structure enables the state variable to record memory effects of normal 
stress variation. For example, a sudden increase of $\sigma$ brings into existence a set of freshly formed asperity contacts, hence reducing the average contact maturity, signaled by a reduction of $\psi$. A sudden decrease of $\sigma$ may rupture the least well adhering contacts, which may generally be expected to be the least mature of the population, and so increases the average maturity, signaled by increase of $\psi$. It may demand too much of a single state variable to represent in a fully realistic way the effects of prior variation of both $V$ and $\sigma$, but that is what is attempted in this formulation.

\subsection{Form for linearized perturbation}

Let the shear stress, normal stress, and slip rate have the respective values $\tau_{0}, \sigma_{0}$, and $V_{0}$ in a state of steady sliding which is to be perturbed. We make the following definitions:

$$
\begin{aligned}
& a=[V \partial F(\sigma, V, \psi) / \partial V] / \sigma, \\
& a-b=\left[V \partial \tau_{\mathrm{ss}}(\sigma, V) / \partial V\right] / \sigma, \\
& f=\partial \tau_{\mathrm{ss}}(\sigma, V) / \partial \sigma, \\
& f-\alpha=\partial F(\sigma, V, \psi) / \partial \sigma-G_{2}(\sigma, V, \psi) \partial F(\sigma, V, \psi) / \partial \psi, \\
& L=V /\left[\partial G_{1}(\sigma, V, \psi) / \partial \psi\right],
\end{aligned}
$$

where, in all functions on the right sides, derivatives are evaluated in the steady state at $\sigma_{0}, V_{0}$, and $\psi_{0}$ with $\psi_{0}=\psi_{\mathrm{ss}}\left(\sigma_{0}, V_{0}\right)$. Note that $f$ here, as well as in Sections 4 and 5 (it does not appear in Section 3), is used in a special way given by the formulae above. In the next two Sections 2.3 and $2.4, f$ is used in its usual meaning to denote the general friction coefficient $f=\tau / \sigma$, although that will coincide with $f$ as defined above if evaluated in steady state. The other notations on the left sides are chosen to coincide with standard notations for parameters in the well-known logarithmic forms of rate and state friction laws, as will be discussed. At this point it is well to emphasize that the results given subsequently, on linearized stability analysis, will just depend on $a, b, f, \alpha, L, \sigma_{0}$, and $V_{0}$, and not further on the detailed form of the functions $F, G_{1}$, and $G_{2}$.

If constitutive laws of the class presented are linearized about steady state, and if explicit reference to the state variable is suppressed, those laws then linearize to

$$
\frac{\mathrm{d} \tau}{\mathrm{d} t}=\frac{a \sigma_{0}}{V_{0}} \frac{\mathrm{d} V}{\mathrm{~d} t}+(f-\alpha) \frac{\mathrm{d} \sigma}{\mathrm{d} t}-\frac{V_{0}}{L}\left[\tau-\tau_{\mathrm{ss}}(\sigma, V)\right],
$$

where, again within the linearization,

$$
\tau_{\mathrm{ss}}(\sigma, V)=\tau_{0}+f\left(\sigma-\sigma_{0}\right)+\frac{(a-b) \sigma_{0}}{V_{0}}\left(V-V_{0}\right) .
$$

Thus $f$ plays the role of the classical friction coefficient relative to steady-state strength, and $(f-\alpha)$ plays a similar role relative to an instantaneous change in normal stress. The parameter $a(>0)$ characterizes the direct (instantaneous) velocity dependence, but steady-state velocity dependence is characterized by $a-b$, which can be positive or negative. Those cases correspond, respectively, to steady-state velocity strengthening or velocity weakening. The latter is of greatest interest for sliding between identical 


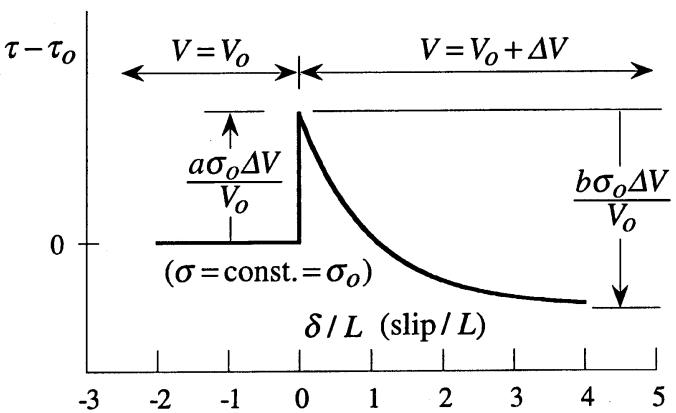

(a)

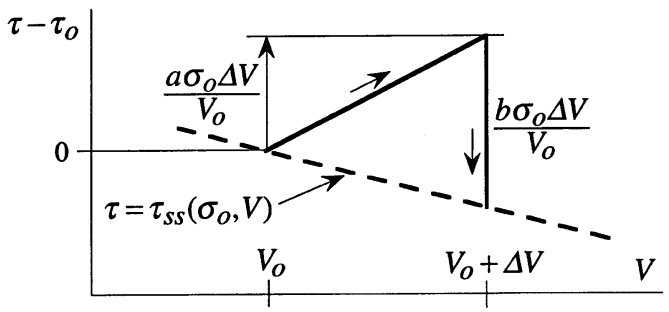

(b)

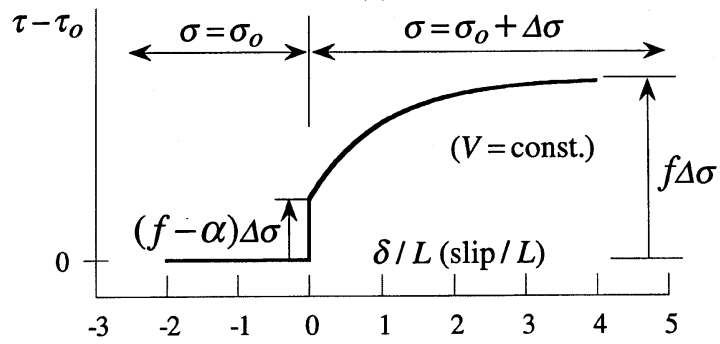

(c)

Fig. 2. Depiction of constitutive response for small perturbations from a state of steady sliding like in Fig. 1: (a) Sudden jump in sliding rate at fixed normal stress; (b) The same, in a stress versus rate plot; (c) Sudden jump in normal stress at fixed sliding rate.

elastic solids, in which case velocity weakening, $b>a$, is required for instability. We will see here how that conclusion generalizes to bi-materials. Finally, $L$ measures a characteristic slip distance over which state evolves in sliding, due to replenishment of the population of asperity contacts. The constitutive response is illustrated in Fig. 2.

The linearized expressions, given above, of single-state variable laws were previously derived by Rice (1983) for the special case $\sigma=$ constant $=\sigma_{0}$. Also, if all velocity dependence of friction is neglected $(a=a-b=0)$ and if $f=\alpha$ so that there is no instantaneous response to normal stress change, then the above expression corresponds to one which Ranjith and Rice (2001) and Cochard and Rice (2000) showed would regularize the ill-posedness of elastodynamic problems of sliding between dissimilar materials mentioned in the introduction. 


\subsection{Physical and empirical basis for standard rate and state laws}

The specific rate and state constitutive relations most often used in the literature have been developed in work by Dieterich $(1979,1981)$ and Ruina (1983). The latter explicitly introduced the concept of state variables. The original work focused on sliding at constant normal stress $\sigma$, writing the friction law in the form

$$
\tau=\sigma f=\sigma\left[f_{*}+a \ln \left(V / V_{*}\right)+\psi\right],
$$

where $f_{*}, a$ and $V_{*}$ are constants, and $f=f_{*}+a \ln \left(V / V_{*}\right)+\psi$ can be called the coefficient of friction. Here $V_{*}$ can be chosen arbitrarily, and if it is arranged in the definition of the state variable that $\psi$ vanishes in steady-state sliding at rate $V_{*}$, then $f_{*}$ represents the friction coefficient for the steady-state sliding with the velocity $V_{*}$. This gives a specific form for $\tau=F(\sigma, V, \psi)$ above and the symbol $a$ is used consistently with the definition for linearization about steady state.

The $\ln \left(V / V_{*}\right)$ term was introduced empirically by Dieterich and Ruina. It is now generally assumed to be descended from an Arrhenius activated rate process describing creep at asperity contacts. That interpretation had been mentioned earlier by Stesky (1977), was implicit in Chester (1994), and was suggested explicitly, in the rate and state framework, by Heslot et al. (1994), Bréchet and Estrin (1994), Baumberger (1997), Sleep (1997), Persson (1998), Baumberger et al. (1999), Lapusta et al. (2000), and Nakatani (2001). In such an interpretation, for the simple case of a single activated process, we write the slip rate as

$$
V=V_{1} \exp \left(-E / k_{\mathrm{B}} T\right)
$$

and understand the activation energy to have the form $E=E_{1}-\tau_{\mathrm{c}} \Omega$, where $\tau_{\mathrm{c}}$ is the average shear stress at asperity contacts and $\Omega$ is the activation volume for the process there. $V_{1}$ can be regarded as an attempt frequency times a slip displacement per successful attempt, and may plausibly be assumed to be at most of order $c_{\mathrm{s}}$, and possibly much less (see below). If $\sigma_{\mathrm{c}}$ is the average normal stress borne at the contacts, then $\tau_{\mathrm{c}} / \sigma_{\mathrm{c}}=\tau / \sigma$. (Note that if $A_{\mathrm{c}}$ is the asperity contact area and $A$ is the nominal area, then $\tau_{\mathrm{c}} A_{\mathrm{c}}=\tau A$ and $\sigma_{\mathrm{c}} A_{\mathrm{c}}=\sigma A$; the ratio of the two equations gives $\tau_{\mathrm{c}} / \sigma_{\mathrm{c}}=\tau / \sigma$.) We can thus write $\tau_{\mathrm{c}}=\sigma_{\mathrm{c}} \tau / \sigma$ and rewrite the activated rate equation as

$$
\tau=\sigma\left[\frac{E_{1}}{\sigma_{\mathrm{c}} \Omega}+\frac{k_{\mathrm{B}} T}{\sigma_{\mathrm{c}} \Omega} \ln \left(\frac{V}{V_{1}}\right)\right] .
$$

That reproduces the empirical logarithmic form of the direct velocity dependence with the interpretation

$$
a=k_{\mathrm{B}} T / \sigma_{\mathrm{c}} \Omega .
$$

If we further assume that $E_{1} / \sigma_{\mathrm{c}} \Omega$ is not a constant, but, rather, varies modestly with changes in the maturity of the contact population (because of local plastic flow processes allowing better atomic scale matching, desorption of trapped impurities in the contact region, etc.), then we can regard $E_{1} / \sigma_{\mathrm{c}} \Omega$ as a state dependent variable, or, equivalently, write it in the form $f_{*}+\psi$ when $V_{*}$ is identified as $V_{1}$, where $\psi$ is a 
state variable. Empirically, it is known that the variations in the state variable part of the strength, in typical experimental settings, is a modest amount of the total strength. That is, the magnitude of variations in $\psi$ are typically quite small compared to $f_{*}$, which signals that $E_{1} / \sigma_{\mathrm{c}} \Omega$ will vary only modestly with state. Of course, the product $\sigma_{\mathrm{c}} \Omega$ entering the denominator of $a$ should also vary with the maturity of a contact so, strictly, it too may vary with the state variable, although that possibility has been neglected in use of the logarithmic form of the friction law. The total variation in $f$ from such state dependence of $a$ must be expected to be a fractionally small change in an already small contribution to $f$, so it is probably a reasonable approximation to treat $a$ as if it was independent of changes in state.

Recent optical measurement of asperity contact sizes (Dieterich and Kilgore, 1994, 1996), in the friction of brittle optically transparent materials (including quartz), suggest that $\sigma_{\mathrm{c}}$ is remarkably large, of order $0.2 \mu$. That is consistent with an estimate by Boitnott et al. (1992) and with microhardness indentation strengths measured independently by Dieterich and Kilgore. (Since typically $\tau_{\mathrm{c}} / \sigma_{\mathrm{c}}=\tau / \sigma \approx 0.6$ for rocks such as granite, that implies that $\tau_{\mathrm{c}}$ is of order $0.1 \mu$ in such systems; $0.1 \mu$ is a standard estimate of the theoretical shear strength, and that suggests an unusual type of local plasticity at the contacts.) Among the most precise measurements of $a$ are probably those of Ruina $(1980,1983)$ on quartzite. He used servo-control based on a displacement measurement very near the slip surface to simulate a very high stiffness apparatus, and thus to impose very abrupt slip rate changes, resulting in $a \approx$ 0.009 at room temperature $\left(293 \mathrm{~K}, k_{\mathrm{B}} T=4.04 \times 10^{-21} \mathrm{~J}\right)$. For granite at room temperature, Linker and Dieterich (1992), using a servo-control system similar to that of Ruina $(1980,1983)$, report $a=0.0104 \pm 0.0007$. That is somewhat higher than the range $a \approx 0.004-0.0075$ given earlier by Tullis and Weeks (1986) from measurements in a less stiff torsional apparatus, and we use $a=0.01$ for granite here. We can thus calculate activation volumes from the formula above for $a$, using $\sigma_{\mathrm{c}} \approx$ $0.2 \mu \approx 6.0 \mathrm{GPa}$ for both materials, and that gives $\Omega \approx 5 \times(0.25 \mathrm{~nm})^{3}$ for quartzite and $4 \times(0.25 \mathrm{~nm})^{3}$ for granite. Those values correspond to a few atomic volumes, and hence provide plausible activation volumes, suggesting merit to this interpretation of the logarithmic law.

Given measured values of $a$, and of $\tau / \sigma$ at any given slip rate $V$, we can estimate the activation energy $E_{1}$, even from data at a single temperature, if we assume a value for $V_{1}$. Fortunately, the exact values chosen for $V$ and $V_{1}$ do not matter very critically for this estimate, because of the logarithm and because of the small value of its factor $a$. $V_{1}$ should be an attempt frequency times the slip of the contact per successful jump. The attempt frequency should be of order of a lattice vibration frequency, hence of order $c_{\mathrm{s}} / d_{0}$, where $d_{0}$ is a lattice spacing. Thus the largest plausible value would seem to be $V_{1} \approx c_{\mathrm{s}}$, which would result if the slip per successful jump is of order $d_{0}$, like expected if a dislocation-like defect sweeps the entire asperity contact area per successful jump at a pinning point. Instead, it is much more likely that the successful jump allows only a small portion $A_{1}$ of the contact area $A_{\mathrm{c}}$ to slip a distance of order $d_{0}$; then the total slip per success is $\left(A_{1} / A_{\mathrm{c}}\right) d_{0}$ and $V_{1}$ is of order $c_{\mathrm{s}} A_{1} / A_{\mathrm{c}}$. For example, if $A_{1}$ has dimensions of a few $\mathrm{nm}$, and $A_{\mathrm{c}}$ of a few $\mu \mathrm{m}$, then $V_{1} \approx 10^{-6} c_{\mathrm{s}}$. We will use $V_{1}=10^{-6} c_{\mathrm{s}}=3 \mathrm{~mm} / \mathrm{s}$ to estimate $E_{1}$; the values to be 
given would increase by about $20 \%$ if we had used the upper bound $V_{1}=c_{\mathrm{s}}$ and decrease by $10 \%$ if we used $V_{1}=10^{-9} c_{\mathrm{s}}$. For quartzite, with $a=0.009$ and $\tau / \sigma=0.54$ (Ruina, 1980, 1983) when $V=1 \mu \mathrm{m} / \mathrm{s}$ in steady state at room temperature, one thus obtains

$$
E_{1} \approx 2.7 \times 10^{-19} \mathrm{~J}=1.7 \mathrm{eV}=170 \mathrm{~kJ} / \mathrm{mol}=0.59 \times \mu(0.25 \mathrm{~nm})^{3} .
$$

Linker and Dieterich (1992) do not report a precise value of $\tau / \sigma$ for granite but only note that it is "about 0.7 ", whereas one can infer values from 0.63 to 0.72 from data they plot showing response to normal stress changes of $10 \%$ or more at $V=1 \mu \mathrm{m} / \mathrm{s}$. Tullis and Weeks (1986) show values which span a comparable range, and average about 0.65 , which we use for $\tau / \sigma$ in this case. That, with $a=0.01$, yields

$$
E_{1} \approx 2.9 \times 10^{-19} \mathrm{~J}=1.8 \mathrm{eV}=180 \mathrm{~kJ} / \mathrm{mol}=0.63 \times \mu(0.25 \mathrm{~nm})^{3}
$$

for granite, very close to the value for quartzite.

The logarithmic law is, of course, not suitable very near to $V=0$. A remedy for that is to recognize that the above argument allows for forward activated jumps only, but not backward ones, which should be equally probable at $\tau=0$. A simple remedy, which regularizes the law at $V=0$, is to include backward jumps by replacing $\exp \left(\tau_{\mathrm{c}} \Omega / k_{\mathrm{B}} T\right)=$ $\exp (\tau / a \sigma)$ with $2 \sinh (\tau / a \sigma)$. Such a procedure, motivated by the thermal activation concept, is in standard use in formulating crustal scale earthquake models with rate and state constitutive laws (e.g., Rice and Ben-Zion, 1996; Ben-Zion and Rice, 1997; Lapusta et al., 2000).

In contrast to the $\ln (V)$ term just discussed, the representation of the state variable has been entirely empirical except for the following concepts: (1) The variable measures the maturity of contacts (more mature being stronger), and (2) Slip over a distance called $L$ here, but often denoted $d_{\mathrm{c}}$, sufficient to replenish the population of asperity contacts, removes memory of prior state. In writing the evolution law for $\psi$, at constant $\sigma$, as $\dot{\psi}=-G(V, \psi)$, the following alternatives are in common use: The DieterichRuina "slip" law (to use the terminology of Perrin et al., 1995) assumes that contact strength matures only during slip, that the evolution from one steady state to another (following a sudden change in slip rate) is exponential in the amount of slip, and that the steady-state strength also varies logarithmically with $V$. Those assumptions correspond to the form

$$
G_{1}^{\mathrm{slip}}(V, \psi)=(V / L)\left[\psi+b \ln \left(V / V_{*}\right)\right],
$$

where $b$ is a constant. The Dieterich-Ruina "ageing" law, or "slowness" law, assumes instead that strength varies with the logarithm of the mean contact lifetime $\theta$, and hence writes $\psi=b \ln \left(V_{*} \theta / L\right)$, again with $b$ being a constant. The evolution law for $\theta$ (which implies one for $\psi$ ) is thus constrained to make $\dot{\theta}=1$, or $\theta=t$, in non-sliding contact, but to make $\theta=L / V$ in steady-state sliding. Those limits are met by writing $\dot{\theta}=1-V \theta / L$ which corresponds to a law for $\dot{\psi}$ given by

$$
G_{1}^{\text {ageing }}(V, \psi)=(b / L)\left[V-V_{*} \exp (-\psi / b)\right] .
$$


Both laws give $\psi_{\mathrm{ss}}=-b \ln \left(V / V_{*}\right)$ and thus give the same strength in steady-state sliding

$$
\tau_{\mathrm{ss}}(\sigma, V)=\sigma\left[f_{*}+(a-b) \ln \left(V / V_{*}\right)\right] .
$$

Hence $f=f_{*}+(a-b) \ln \left(V / V_{*}\right)$ is the steady-state coefficient of friction.

\subsection{Response to variable normal stress}

In attempting to represent the effects on state of variable normal stress, Linker and Dieterich (1992) tried to maintain as much of this framework as possible, wishing to continue to understand that $\tau=F(\sigma, V, \psi)$ with $F$ given by the logarithmic law. Since their experiments showed fading memory of effects of alteration of normal stress, that forced them to assume that $\psi$ varies not only with slip and time as in the two formulations above, but also with $\sigma$. (As remarked, it is an approximation to assume that a single-state variable adequately characterizes state; its validity may be even less secure when we include the effects of variable normal stress.) They therefore proposed to fit their experiments to a law of the form

$$
\dot{\psi}=-G_{1}(V, \psi)-\alpha \dot{\sigma} / \sigma
$$

where $G_{1}(V, \psi)$ is one of the two forms just discussed. They actually did that relative to the Dieterich-Ruina ageing form but, as Perfettini et al. (2001) discuss, the procedure they used could be implemented with either law. Thus the function $G_{2}(\sigma, V, \psi)$ introduced earlier corresponds to $\alpha / \sigma$. Linker and Dieterich regarded $\alpha$ as a constant, although Perfettini et al. (2001) and Cochard and Rice (2000) allow the possibility that $\alpha=\alpha(V, \psi)$.

This new law retains the same form for $\psi_{\mathrm{ss}}$, as $\psi_{\mathrm{ss}}=-b \ln \left(V / V_{*}\right)$, and the expression for $\tau_{\mathrm{ss}}(\sigma, V)$ and $f$ in steady state, as given above, remain valid. However, for changes of $\sigma$ and $V$, one has

$$
\begin{aligned}
\dot{\tau} & =\frac{\mathrm{d}}{\mathrm{d} t}(f \sigma)=f \dot{\sigma}+\sigma\left(\frac{a}{V} \dot{V}+\dot{\psi}\right)=f \dot{\sigma}+\sigma\left[\frac{a}{V} \dot{V}-G_{1}-\frac{\alpha}{\sigma} \dot{\sigma}\right] \\
& =[f(V, \psi)-\alpha(V, \psi)] \dot{\sigma}+\frac{a \sigma}{V} \dot{V}-\sigma G_{1}(V, \psi) .
\end{aligned}
$$

This shows that if $V$ is held constant but $\sigma$ is suddenly altered (sudden enough that we can neglect the $\sigma G_{1}$ term in calculating the resulting change in $\tau$ ), then

$$
\mathrm{d} \tau=[f(V, \psi)-\alpha(V, \psi)] \mathrm{d} \sigma \quad \text { with } \mathrm{d} \psi=-\alpha(V, \psi) \mathrm{d} \sigma / \sigma .
$$

The integrals of that set of expressions, over the finite change in $\sigma$, provide the corresponding finite change in $\tau$. Hence $f-\alpha$ is the parameter which acts like a coefficient of friction during a sudden alteration of normal stress at fixed $V$.

As Perfettini et al. (2001) and Cochard and Rice (2000) discuss, the particular choice $\alpha(V, \Psi)=f(V, \Psi)$ assures that there is precisely zero instantaneous alteration of $\tau$ in response to an instantaneous alteration of $\sigma$. Such a response of $\tau$, surprising as it is, is what was found in the oblique shock wave experiments of Prakash and Clifton (1992) and Prakash (1998). Those involved plates of hard steel or titanium alloy 
sliding against a plate of WC-Co cutting-tool material. Following a step decrease of $\sigma$ on the sliding surface, achieved by arrival of a reflected shock wave, $\tau$ was found to evolve continuously (over a few $\mu \mathrm{m}$ slip, or few tenths of $\mu$ s time; it is not clear from the experiment which is the better description) to its new value associated with the new $\sigma$. Ranjith and Rice (2001) noted that such a constitutive feature would regularize the otherwise ill-posed problem of dynamic sliding between dissimilar half-spaces with a constant coefficient of friction. However, those experiments involved $V$ of order 1 to $10 \mathrm{~m} / \mathrm{s}$. They lead to a different results than do experiments (Linker and Dieterich, 1992; Richardson and Marone, 1999) at slip rates of order $10^{-6}$ slower, on rock materials. Studies of normal stress changes at those lower rates have led to the conclusion that $\alpha$ is of the order of $f / 3$. Further, studies on the effects of high-frequency oscillations of $\sigma$ during the slow sliding of a polymer system led Bureau et al. (2000) to conclude that use of the $\alpha \dot{\sigma} / \sigma$ term in $\dot{\psi}$ does not enable a good fit to their experiments. Thus there is much yet to be understood on representing memory effects of normal stress variation.

\section{Frictional sliding of identical solids and relation of quasi-static and dynamic analyses}

Before progressing to the dissimilar materials case, we consider, in the context of elastodynamics, the stability of the steady sliding of two identical half-spaces on one another. We use rate and state friction with positive direct velocity effect $(a>0)$, and show that then the problem of finding the response to perturbation of steady sliding is well-posed for all sliding velocities, in that the perturbations of the shortest wavelengths (highest wavenumbers) are always stable. (That is shown in a more general way, applicable to dissimilar materials too, in Section 5.) For slow enough sliding velocities, we show that the response to perturbations becomes quasi-static and converges to the solution of the corresponding quasi-static problem. We also demonstrate how compromises of the rate and state constitutive framework, in the direction of classical friction laws, prevent the problem of response to perturbation from having a quasi-static range, even at slow sliding velocities, and may lead to prediction of paradoxical features like supersonic propagation of slip perturbations, or even to ill-posedness.

\subsection{Response to anti-plane perturbations}

To begin with a simple case, we consider anti-plane perturbations of an $\exp (\mathrm{i} k x)$ space dependence, where now the $x$-axis lies in the interface and is directed perpendicular to the common direction of slip and shear loading, which is the $z$ direction (unlike in Fig. 1, which has been drawn for the case of in-plane perturbations considered in Sections 3.3, 3.4, 4 and 5). This problem of linear perturbation was partially addressed by Rice and Ruina (1983). The sliding occurs on the plane $y=0$ and we write the displacement field as

$$
u_{z}(x, y, t)=\frac{1}{2} V_{0} t \operatorname{sign}(y)+\hat{u}_{z}(x, y, t)
$$


where $\hat{u}_{z}$ is the perturbed part and satisfies $c_{\mathrm{s}}^{2} \nabla^{2} \hat{u}_{z}=\partial^{2} \hat{u}_{z} / \partial t^{2}$. The perturbation of shear stress and sliding velocity at the interface are then

$$
\begin{gathered}
\tau(x, t)-\tau_{0}=\left.\mu \frac{\partial \hat{u}_{z}(x, y, t)}{\partial y}\right|_{y=0^{+}}=\left.\mu \frac{\partial \hat{u}_{z}(x, y, t)}{\partial y}\right|_{y=0^{-}}, \\
V(x, t)-V_{0}=\frac{\partial \hat{u}_{z}\left(x, 0^{+}, t\right)}{\partial t}-\frac{\partial \hat{u}_{z}\left(x, 0^{-}, t\right)}{\partial t} .
\end{gathered}
$$

There is no perturbation of normal stress $\sigma_{0}$, and the perturbations of $\tau$ and $V$ must therefore satisfy, within linearization

$$
\frac{\partial \tau}{\partial t}=\frac{a \sigma_{0}}{V_{0}} \frac{\partial V}{\partial t}-\frac{V_{0}}{L}\left[\tau-\tau_{0}-(a-b) \frac{V-V_{0}}{V_{0}}\right] .
$$

Looking for a solution with $x$ and $t$ dependence as $\exp (\mathrm{i} k x+p t)$, where $k$ is real, one solves the wave equation with

$$
\hat{u}_{z}=\frac{1}{2} A \operatorname{sign}(y) \exp \left[\mathrm{i} k x-\left(k^{2}+p^{2} / c_{\mathrm{s}}^{2}\right)^{1 / 2}|y|+p t\right],
$$

where $A$ is a constant and the square-root term is cut from $\mathrm{i}|k| c_{\mathrm{s}}$ to $\mathrm{i} \infty$, and from $-\mathrm{i} \infty$ to $-\mathrm{i}|k| c_{\mathrm{s}}$, in the complex $p$ plane to assure that it has a non-negative real part. (For a quasi-static model, in which the displacement satisfies $\nabla^{2} \hat{u}_{z}=0$, we replace $\left(k^{2}+p^{2} / c_{\mathrm{s}}^{2}\right)^{1 / 2}$ with $|k|$ here. $)$

Thus the stress and velocity perturbations are

$$
\begin{aligned}
& \tau(x, t)-\tau_{0}=-\frac{1}{2} \mu A\left(k^{2}+p^{2} / c_{\mathrm{s}}^{2}\right)^{1 / 2} \exp (\mathrm{i} k x+p t), \\
& V(x, t)-V_{0}=p A \exp (\mathrm{i} k x+p t) .
\end{aligned}
$$

The first expression is $\tau(x, t)-\tau_{0}=-(1 / 2) \mu A|k| \exp (\mathrm{i} k x+p t)$ in the quasi-static model. One then finds that in order for $\tau$ and $V$ to meet the above-linearized frictional constitutive relation, $p$ must satisfy

$$
\frac{\mu}{2}\left(p+\frac{V_{0}}{L}\right)\left(k^{2}+\frac{p^{2}}{c_{\mathrm{s}}^{2}}\right)^{1 / 2}+\frac{\sigma_{0} p}{V_{0}}\left[a p-(b-a) \frac{V_{0}}{L}\right]=0 .
$$

For the quasi-static model, the corresponding expression is

$$
\frac{\mu}{2}\left(p+\frac{V_{0}}{L}\right)|k|+\frac{\sigma_{0} p}{V_{0}}\left[a p-(b-a) \frac{V_{0}}{L}\right]=0 .
$$

It may be shown (Rice and Ruina, 1983) in both cases that for large $|k|$ (short wavelengths) one has $\operatorname{Re}(p)<0$ and hence stability, assuming $a>0$. However, as $|k|$ is reduced in magnitude, one finds that for velocity-weakening surfaces (i.e., surfaces with $b-a>0)$, a value $|k|=k_{\mathrm{cr}}$ is ultimately reached at which stability is lost. That occurs because $\operatorname{Re}(p)$ changes sign, as a pair of complex-conjugate roots traverse the $\operatorname{Im}(p)$ axis. This is a Hopf bifurcation, and it occurs at the critical wavenumber

$$
k_{\mathrm{cr}} \equiv \frac{2 \pi}{\lambda_{\mathrm{cr}}}=\frac{2(b-a) \sigma_{0}}{\mu L} \sqrt{1+q^{2}},
$$


where here and subsequently, $q$ denotes a dimensionless measure of the unperturbed sliding velocity, given by

$$
q=\mu V_{0} /\left[2 \sqrt{a(b-a)} \sigma_{0} c_{\mathrm{s}}\right]
$$

The corresponding quasi-static result is (Rice and Ruina, 1983)

$$
k_{\mathrm{cr}} \equiv \frac{2 \pi}{\lambda_{\mathrm{cr}}}=\frac{2(b-a) \sigma_{0}}{\mu L} .
$$

If we write the space-time dependence at the critical wavenumbers $\left(k=k_{\mathrm{cr}}\right.$ and $\left.k=-k_{\mathrm{cr}}\right)$ as $V(x, t)-V_{0}=A \exp \left[ \pm \mathrm{i} k_{\mathrm{cr}}(x \pm c t)\right]$, so that $c$ is the phase velocity of the neutrally propagating modes, then

$$
c=q c_{\mathrm{s}} / \sqrt{1+q^{2}}=\left[\mu V_{0} / 2 \sqrt{a(b-a)} \sigma_{0}\right] / \sqrt{1+q^{2}} .
$$

That speed is always subsonic, i.e., satisfies $c<c_{\mathrm{s}}$. The corresponding quasi-static solution is $c=\left[\mu V_{0} / 2 \sqrt{a(b-a)} \sigma_{0}\right]$.

It is interesting to notice that at the critical wavenumber, time-dependence of the response to perturbation is

$$
\exp (p t)=\exp \left( \pm \mathrm{i} k_{\mathrm{cr}} c t\right)=\exp \left( \pm \mathrm{i} \sqrt{\frac{b-a}{a}} \frac{V_{0} t}{L}\right) .
$$

That has no dependence on the inertial $\left(c_{\mathrm{s}}\right)$ or even elastic $(\mu)$ properties of the sliding solids, and is the same in both the dynamic and quasi-static analyses. We will further show that this expression holds for in-plane perturbations as well.

We see that the results of the elastodynamic analysis acquire quasi-static character for small enough sliding velocities $0<q \ll 1$ or

$$
V_{0} \ll\left[2 \sqrt{a(b-a)} \sigma_{0} c_{\mathrm{s}}\right] / \mu
$$

because, in the limit $q \rightarrow 0, k_{\mathrm{cr}}$ becomes independent of the (dynamic) parameter $c_{\mathrm{s}}$, and $c / c_{\mathrm{s}}=q / \sqrt{1+q^{2}} \rightarrow 0$, so that for $q \ll 1$ neutrally stable modes propagate much slower than the shear wave speed. Moreover, for $q \ll 1$, the elastodynamic response corresponds to the solution of the corresponding quasi-static problem. For granite at room temperature, it is typically the case that $\sqrt{a(b-a)}$ is of order 0.005 (e.g., Tullis and Weeks, 1986), and thus $q \approx\left[V_{0} /(\mathrm{mm} / \mathrm{s})\right] \times\left(\mathrm{MPa} / \sigma_{0}\right)$. Note also that even though, for sufficiently small $V_{0}$, the speed $c$ satisfies $c \ll c_{\mathrm{s}}$, it will typically still be much larger than $V_{0}$ (by a factor of order $3 \times 10^{6} \mathrm{MPa} / \sigma_{0}$ for granite).

For conclusions from the dynamic analysis to reduce to those of the quasi-static analysis at sufficiently slow slip rates, it is essential that $a>0$, which is what is observed experimentally. The case $a=0$ will be considered in the next section. However, even from the elastodynamic results of this section it is easy to see that when $a \rightarrow 0$, then $q \rightarrow \infty$ for any fixed $V_{0}$, so that we have $k_{\mathrm{cr}} \rightarrow \infty$, which signals that the highest wavenumbers (the shortest wavelengths) become unstable, and $c \rightarrow c_{\mathrm{s}}$, which hints that these highest wavenumbers have phase velocities of the order of the shear wave speed. That suggests that the problem with $a=0$ no longer has a quasi-static range and could even be ill-posed. 


\subsection{Importance of rate and state features for existence of quasi-static range; response to anti-plane perturbations}

We may now see that dynamic analyses using versions of the friction law which neglect all or some of the rate and state features lead to solutions which have no range of quasi-static response to perturbation, no matter how small is (the positive) $V_{0}$ or how large is $|k|$.

Consider first classical friction, in which there are no rate or state effects. We can represent that case by letting all of $a, b$, and $L$ approach zero (after multiplying through by $L / V_{0}$ in the equation for $\left.p\right)$. One then obtains $\left(k^{2}+p^{2} / c_{\mathrm{s}}^{2}\right)^{1 / 2}=0$ which has only the roots $p= \pm \mathrm{i} k c_{\mathrm{s}}$, corresponding to propagation at the shear speed. (Clearly, since $\tau(x, t)$ is constant in this constitutive limit, no matter how $V(x, t)$ changes, so long as it stays positive, the interface will propagate perturbations as shear waves.) Thus there is no quasi-static version of the problem of response to perturbation in this case.

Now consider again the rate and state formulation but simply take $a=0$. (The normal contact law of Oden and Martins, 1985, if interpreted with $\psi$ depending on normal penetration, and the actual normal motion neglected in the dynamics, would fall into such $a=0$ class.) Since the state evolution slip distance $L>0$, the strength $\tau$ then undergoes no change upon an instantaneous change in velocity, but changes only as part of the evolution towards the new steady-state strength. Introducing the notation $P=p /|k| c_{\mathrm{s}}$,

$$
\left(P+\frac{V_{0}}{|k| L c_{\mathrm{s}}}\right)\left(1+P^{2}\right)^{1 / 2}-\frac{2 b \sigma_{0}}{|k| L \mu} P=0 .
$$

At high wavenumbers, $|k| L \gg 1$, and at considerably less high wavenumbers in slow sliding $\left(V_{0} \ll c_{\mathrm{S}}\right)$ with $b \sigma_{0} \ll \mu$ (typically the case), this equation has a solution for which $\left(1+P^{2}\right)^{1 / 2}$ differs from zero by a number of magnitude $\ll 1$. Thus there are roots $P$ in the near vicinity of $\pm i$, implying that the response to perturbation propagates at a speed near $c_{\mathrm{s}}$, so that there is again no quasi-static version of the problem. Also, further analysis shows that there are roots with $\operatorname{Re}(p)>0$ for all values of $k$, so this model does not have the short-wavelength stability assured when $a=0$.

A more extreme compromise of rate and state dependent friction is to let $L \rightarrow 0$. In that limit, $a$ (as distinct from $b-a$ ) becomes an irrelevant variable and the stress $\tau$ always corresponds to the steady-state strength $\tau_{\mathrm{ss}}\left(\sigma_{0}, V\right)$, towards which it now evolves instantaneously upon any change of $V$. That is, we are then assuming purely rate-dependent friction which follows the velocity-weakening law $\tau=\tau_{\mathrm{ss}}\left(\sigma_{0}, V\right)$, without memory or state dependence. Using the notation

$$
T=-\frac{\partial \tau_{\mathrm{ss}}\left(\sigma_{0}, V\right) / \partial V}{\mu / 2 c_{\mathrm{s}}}=\frac{2(b-a) \sigma_{0} c_{\mathrm{s}}}{\mu V_{0}}
$$

(not to be confused with $T$ used for temperature earlier), and assuming $T>0, p$ is determined in that limit by

$$
\left(1+P^{2}\right)^{1 / 2}-T P=0 .
$$


That provides a problematic model. If $0<T<1, P=p /|k| c_{\mathrm{S}}= \pm \mathrm{i} / \sqrt{1-T^{2}}$. Since $1 / \sqrt{1-T^{2}}>1$, that corresponds to supersonic propagation of perturbations along the interface at phase velocity

$$
c=c_{\mathrm{s}} / \sqrt{1-T^{2}}>c_{\mathrm{s}} .
$$

Since that $c$ is independent of $k$, every Fourier component of a generic perturbation propagates at the same speed, and hence the perturbation leads to a pair of pulses, both propagating supersonically, in opposite directions along the interface. That is a type of propagation in pure velocity-weakening friction which Weertman (1969), and also Knopoff and Landoni (1998), had previously noted. (For in-plane, versus anti-plane, perturbations of slip between identical solids, the propagation speed is supersonic relative to $c_{p}$, unless $T$ is near 0 , as will be discussed in Section 3.4.) If the velocity weakening is stronger, so that $T>1$, then the solutions for $p$ are real,

$$
P=p /|k| c_{\mathrm{s}}= \pm 1 / \sqrt{T^{2}-1} .
$$

While the solution in response to an individual $\exp (\mathrm{i} k x)$ is thus well defined, at least until the exponential growth becomes so large that $V$ turns negative, this solution for $p$ signals an ill-posed model. This is much like has been discussed for the dissimilar material case (Renardy, 1992; Simões and Martins, 1998; Cochard and Rice, 2000; Ranjith and Rice, 2001), in which a similar $p \propto|k|$ occurs, and the problem of response to a generic perturbation at $t=0$, whose spatial Fourier transform falls off less rapidly with $|k|$ than exponentially at large $|k|$, fails to have a solution for $t>0$.

\subsection{Response to in-plane perturbations}

To address in-plane perturbations of slip, we interpret $x$ in $\exp (\mathrm{i} k x)$ as a coordinate axis in the interface along the direction of slip (Fig. 1). Then the perturbed displacement field $\hat{u}_{x}, \hat{u}_{y}$ corresponds to a state of plane strain in the $x, y$ plane, with $u_{y}$ as well as $\sigma\left(=-\sigma_{y y}\right)$ and $\tau\left(=\sigma_{y x}\right)$, but not $u_{x}$, being continuous at $y=0$. If $\hat{u}_{x}= \pm(A / 2) \exp (\mathrm{i} k x+$ $p t$ ) on the two sides of the interface, then the slip velocity perturbation is

$$
V(x, t)-V_{0}=\partial \hat{u}_{x}\left(x, 0^{+}, t\right) / \partial t-\partial \hat{u}_{x}\left(x, 0^{-}, t\right) / \partial t=p A \exp (\mathrm{i} k x+p t)
$$

like in the anti-plane case. Stress perturbations can be obtained by solving the elastodynamic plane strain equations for $\hat{u}_{x}(x, y, t)$ and $\hat{u}_{y}(x, y, t)$, and calculating the associated stresses. The results can be taken from Geubelle and Rice (1995), and show that the perturbation of shear stress is

$$
\begin{aligned}
& \tau(x, t)-\tau_{0}=-\frac{1}{2} \mu|k| A \hat{F}(p) \exp (\mathrm{i} k x+p t), \quad \text { where } \hat{F}(p)=\frac{4 \alpha_{\mathrm{s}} \alpha_{p}-\left(1+\alpha_{s}^{2}\right)^{2}}{\alpha_{\mathrm{s}}\left(1-\alpha_{\mathrm{s}}^{2}\right)}, \\
& \alpha_{\mathrm{s}}=\left(1+p^{2} / k^{2} c_{\mathrm{s}}^{2}\right)^{1 / 2}, \quad \alpha_{p}=\left(1+p^{2} / k^{2} c_{p}^{2}\right)^{1 / 2}
\end{aligned}
$$

Here, as in the anti-plane case, $\alpha_{\mathrm{s}}$ is cut from $\mathrm{i}|k| c_{\mathrm{s}}$ to $\mathrm{i} \infty$ and $-\mathrm{i} \infty$ to $-\mathrm{i}|k| c_{\mathrm{s}}$ in the complex $p$ plane, and $\alpha_{p}$ from $\mathrm{i}|k| c_{p}$ to $\mathrm{i} \infty$ and $-\mathrm{i} \infty$ to $-\mathrm{i}|k| c_{p}$, to assure that they 
have non-negative real parts. The numerator of $\hat{F}(p)$ vanishes at $p= \pm \mathrm{i}|k| c_{\mathrm{R}}$, where $c_{\mathrm{R}}$ is the Rayleigh speed.

The corresponding quasi-static analysis leads to

$$
\tau(x, t)-\tau_{0}=-\frac{\mu}{2(1-v)}|k| A \exp (\mathrm{i} k x+p t),
$$

where $v$ is the Poisson ratio; the dynamic result reduces to that when $p / k c_{\mathrm{s}} \rightarrow 0$ and $p / k c_{p} \rightarrow 0$, since $2\left(c_{p}^{2}-c_{\mathrm{s}}^{2}\right) / c_{p}^{2}=1 /(1-v)$. Thus all results for quasi-static in-plane perturbations are identical to those given earlier for quasi-static anti-plane perturbations, except that $\mu$ of the latter case is now replaced by $\mu /(1-v)$.

To continue with the dynamic in-plane analysis, the linearized constitutive law between $\tau$ and $V$ leads to

$$
\frac{\mu}{2}\left(p+\frac{V_{0}}{L}\right)|k| \hat{F}(p)+\frac{\sigma_{0} p}{V_{0}}\left[a p-(b-a) \frac{V_{0}}{L}\right]=0
$$

as the equation determining $p$, where we are assuming, as always, that $a>0$ and are considering velocity-weakening surfaces so that $b-a>0$. Like for the anti-plane case, large $|k|$ values (short wavelengths) have $\operatorname{Re}(p)<0$ and hence stability, because $a>0$. To find the critical wavenumber at which stability is lost, we observe, like in Rice and Ruina (1983), that roots cannot cross from $\operatorname{Re}(p)<0$ to $\operatorname{Re}(p)>0$ at any $|k|>0$ by passing through $p=0$. Thus we identify $k_{\mathrm{cr}}$ by seeking conditions for there to be roots in the form $p=\mathrm{i}|k| c$, where $c$ is real; $k_{\text {cr }}$ will be the largest $|k|$ at which such roots occur. In that case, $\hat{F}(p)$ depends only on $c$, and we will replace the notation $\hat{F}(p)$ with $F(c)$, where

$$
F(c)=\frac{4 \sqrt{1-c^{2} / c_{\mathrm{s}}^{2}} \sqrt{1-c^{2} / c_{p}^{2}}-\left(2-c^{2} / c_{\mathrm{s}}^{2}\right)^{2}}{c^{2} / c_{\mathrm{s}}^{2} \sqrt{1-c^{2} / c_{\mathrm{s}}^{2}}} .
$$

We examine roots for three cases, $|c|<c_{\mathrm{s}}, c_{\mathrm{s}}<|c|<c_{p}$, and $c_{p}<|c|$, and then summarize the results in Figs. 3 and 4. It is straightforward to see that roots, when they exist, will occur in conjugate pairs $p= \pm \mathrm{i}|k| c$, so that we henceforth assume $c>0$.

In the subsonic regime $c<c_{\mathrm{s}}$, we find that for each value of $q$, the dimensionless unperturbed sliding velocity introduced in Section 3.1, there is a unique value of the phase velocity $c$, and that value is sub-Rayleigh, $0<c<c_{\mathrm{R}}$. To demonstrate that, we note that $F(c)$ is purely real for $c<c_{\mathrm{s}}$ and, separating the real and imaginary parts in the equation determining $p$, we get

$$
\frac{V}{L} \frac{\mu}{2} F(c)=\frac{a \sigma_{0}}{V}|k| c^{2} \quad \text { and } \quad \frac{\mu|k|}{2} F(c)=\frac{(b-a) \sigma_{0}}{L} .
$$

From either of these equations, we see that $F(c)$ has to be positive, and hence potential roots are restricted to sub-Rayleigh phase velocities, $0<c<c_{R}$. Taking the ratio of the two equations, we obtain

$$
|k| c=\frac{V}{L} \sqrt{\frac{b-a}{a}}
$$




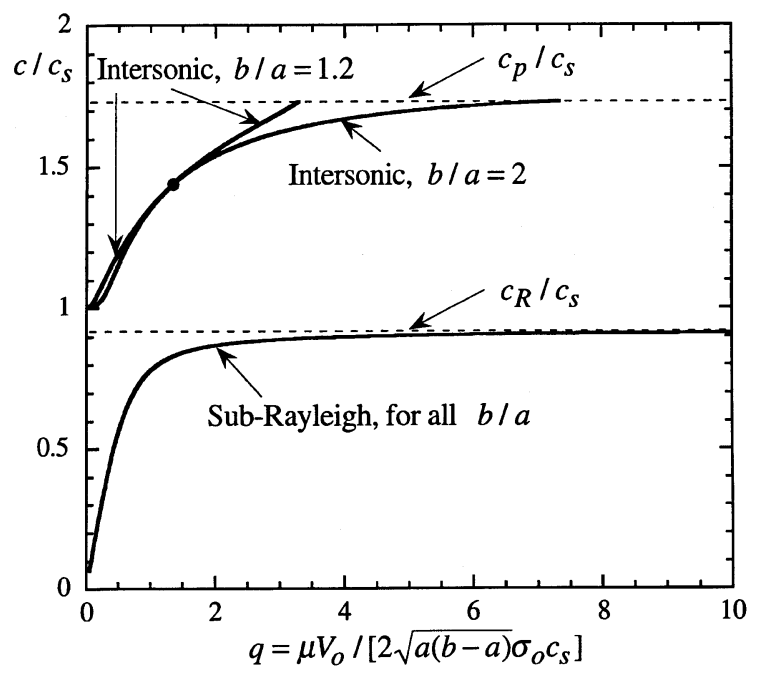

Fig. 3. For in-plane exp $(\mathrm{i} k x+p t)$ perturbations of steady sliding between identical elastic half-spaces. Phase speeds $c$, as function of sliding velocity, are shown when roots cross the $\operatorname{Im}(p)$ axis at $p= \pm \mathrm{i}|k| c$. The sub-Rayleigh branch, which is the same for all ratios $b / a>1$, is shown in Fig. 4 to correspond to the critical wave number, $k_{\mathrm{cr}}$.

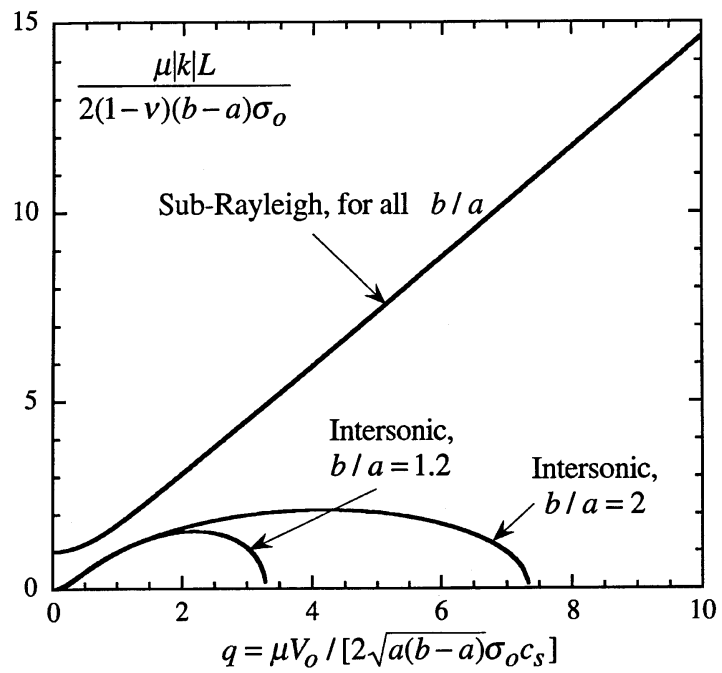

Fig. 4. Wavenumber magnitudes $|k|$, for in-plane $\exp (\mathrm{i} k x+p t)$ perturbations of steady sliding between identical elastic half-spaces, when roots $p$ cross the $\operatorname{Im}(p)$ axis. The branch with sub-Rayleigh phase velocity $c$ (Fig. 3) gives the largest such $|k|$, which therefore corresponds to the critical wave number, $k_{\mathrm{cr}}$. Response to perturbation is stable when $|k|>k_{\mathrm{cr}}$, but unstable when $|k|<k_{\mathrm{cr}}$. 
which is the same result as for the critical value of $|k| c$ in the anti-plane case. Substituting the last result into the second equation produces the following equation for determining $c$ :

$$
\frac{c / c_{\mathrm{s}}}{F(c)}=\frac{\mu V}{2 \sqrt{a(b-a)} \sigma_{0} c_{\mathrm{s}}} \equiv q .
$$

Since $F(c)$ decreases monotonically with increasing $c$, and passes through 0 at $c_{\mathrm{R}}$, for each value of $q$ (positive by definition), this equation has a unique root $c$ with $0<c<c_{\mathrm{R}}$. The corresponding wavenumber $|k|$, referred to as $\left|k_{\mathrm{sub}}\right|$ below, can then be found from the expression for $|k| c$.

In the intersonic regime $c_{\mathrm{s}}<c<c_{p}$, more solutions of the form $p= \pm \mathrm{i}|k| c$ exist, for each $b / a>1$ and for small enough $q$. In this case $F(c)$ has nonzero real and imaginary parts (except for the Eshelby value $c=\sqrt{2} c_{\mathrm{s}}$, for which $F(c)$ is purely real, and the results cited above apply). We write $F(c)=F_{1}(c)+\mathrm{i} F_{2}(c)$, where $F_{1}$ and $F_{2}$ (real) are evaluated approaching the branch cuts from the right half-plane $\operatorname{Re}(p)>0$. Separating the real and imaginary parts in the equation determining $p$, we find that

$$
F_{1}(c)-r F_{2}(c)=\frac{2 a \sigma_{0} c_{\mathrm{s}}}{\mu V} \frac{c}{c_{\mathrm{s}}} r \quad \text { and } \quad r F_{1}(c)+F_{2}(c)=\frac{2(b-a) \sigma_{0} c_{\mathrm{s}}}{\mu V} \frac{c}{c_{\mathrm{s}}},
$$

where $r=|k| c L / V$. Inspecting the formula for $F(c)$, we observe that $F_{1}>0$ and $F_{2}>0$. The above equations give a quadratic equation for $r$ in the form

$$
r^{2}+r F_{2} b /\left(F_{1} a\right)-(b-a) / a=0,
$$

which has only one positive solution

$$
r \equiv \frac{|k| c L}{V}=-\frac{b F_{2}(c)}{2 a F_{1}(c)}+\sqrt{\left[\frac{b F_{2}(c)}{2 a F_{1}(c)}\right]^{2}+\frac{b-a}{a}} .
$$

Then, from the second equation of the set above, we get the following equation to determine the intersonic roots $c$ (when they exist)

$$
\begin{aligned}
& \frac{\sqrt{b / a-1} c / c_{\mathrm{s}}}{\sqrt{\left[F_{2}(c) b /(2 a)\right]^{2}+(b / a-1) F_{1}^{2}(c)-F_{2}(c) b /(2 a)+F_{2}(c)}} \\
& =\frac{\mu V}{2 \sqrt{a(b-a)} \sigma_{0} c_{\mathrm{s}}} \equiv q .
\end{aligned}
$$

We denote the left-hand side of the above equation by $G\left(b / a, c / c_{\mathrm{s}}\right)$ and observe that the equation has solutions for each value of $b / a>1$ only when $q<G\left(b / a, c_{p} / c_{\mathrm{S}}\right)$. The corresponding wavenumber $|k|$, called $\left|k_{\text {int }}\right|$ below, is then determined by the expression for $r$ above. No intersonic solutions exist if $q>G\left(b / a, c_{p} / c_{\mathrm{s}}\right)$.

Finally, it can be easily shown that no solutions exist in the supersonic regime $c_{p}<c$.

To sum it up, we have been seeking values of $|k|$ and $c(>0)$ at which roots pass from $\operatorname{Re}(p)<0$ to $\operatorname{Re}(p)>0$ through the points $p= \pm \mathrm{i}|k| c$. We showed that for each value $q>0$ of the dimensionless sliding velocity, a solution $\left|k_{\text {sub }}\right|$ exists with 
sub-Rayleigh phase velocity $0<c_{\text {sub }}<c_{\mathrm{R}}$. In addition, for a limited range of $q$, given by $q<G\left(b / a, c_{p} / c_{\mathrm{s}}\right)$, there exist additional solutions $\left|k_{\text {int }}\right|$, one for each $b / a>1$, with intersonic phase velocity $c_{\mathrm{s}}<c_{\text {int }}<c_{p}$. From the above expressions for $\left|k_{\text {sub }}\right|$ and $\left|k_{\text {int }}\right|$, and the trivial relation $c_{\text {sub }}<c_{\mathrm{s}}<c_{\text {int }}$, we get

$$
\left|k_{\text {int }}\right|<\frac{V}{c_{\text {int }} L} \sqrt{\frac{b-a}{a}}<\frac{V}{c_{\text {sub }} L} \sqrt{\frac{b-a}{a}}=\left|k_{\text {sub }}\right| .
$$

So, for each given $q$, the sub-Rayleigh critical wavenumber $\left|k_{\text {sub }}\right|$ is either the only one existing, or the largest one. Thus $\left|k_{\text {sub }}\right|$ is the critical wavenumber, $k_{\mathrm{cr}}$, at which the stability is lost. Figs. 3 and 4 illustrate the dependence of the two wavenumber solutions and their phase velocities on the parameters $q$ and $b / a$. Like for the anti-plane case, the condition $a>0$ assures that results of the dynamic analysis reduce to those of the quasi-static analysis at sufficiently slow sliding velocities.

In Section 4, quasi-static stability results for in-plane perturbation of dissimilar materials are considered. In the limit case of identical materials, they will have to agree with the quasi-static results of this sub-section. It is only the in-plane perturbations, and not the anti-plane ones, which could be qualitatively different in the dissimilar material case (and hence are of interest), because they involve coupling of slip perturbation to alteration of normal stress.

\subsection{Importance of rate and state features; response to in-plane perturbations}

We showed in Section 3.2, for anti-plane perturbations, how compromises from the rate and state framework, in the direction of classical friction laws, may lead to lack of existence of a quasi-static response range and to other paradoxical features. A similar discussion may be repeated for the case of in-plane perturbations. However, we confine our attention here just to classical laws of pure velocity-dependent friction, of velocity-weakening type. In that case, the time dependence is determined by

$$
\hat{F}(p)-T p /\left(|k| c_{\mathrm{s}}\right)=0,
$$

where $\hat{F}(p)$ is defined in Section 3.3 and $T$ is defined in Section 3.2. This shares features of the anti-plane case. Consider first strong velocity weakening, so that $T>1$. Then, since $\hat{F}(0)=1 /(1-v)$ and $\hat{F}(p) \rightarrow p /|k| c_{\mathrm{s}}$ as $p \rightarrow \infty$ in $\operatorname{Re}(p)>0$, we are assured that for every such $T$, there exists a real-positive solution for $p /|k| c_{\mathrm{S}}$. Fig. 5 plots $\hat{F}(p)$ and $T p /|k| c_{\mathrm{s}}$ against $p /|k| c_{\mathrm{s}}$ along the positive real axis (for $c_{p}=\sqrt{3} c_{\mathrm{s}}$ ) and shows graphically the origin of the result. That means that $p \propto|k|$ and suggests ill-posedness of the model, just as in the anti-plane case for $T>1$. Also, like in that case, the factor of $|k|$ diverges as $T \rightarrow 1^{+}$.

Now consider weaker velocity weakening, $T<1$. There is no longer a solution on the $\operatorname{Re}(p)$ axis but, so long as $T$ is not so near 0 (i.e., for $T_{\min }<T<1$, where $T_{\min }$ is to be defined below), there are solutions of the type $p= \pm \mathrm{i}|k| c$ where $c$ is real and positive. In the notation of Section 3.3, such solutions would have to satisfy

$$
F(c)-\mathrm{i} T c / c_{\mathrm{s}}=0
$$




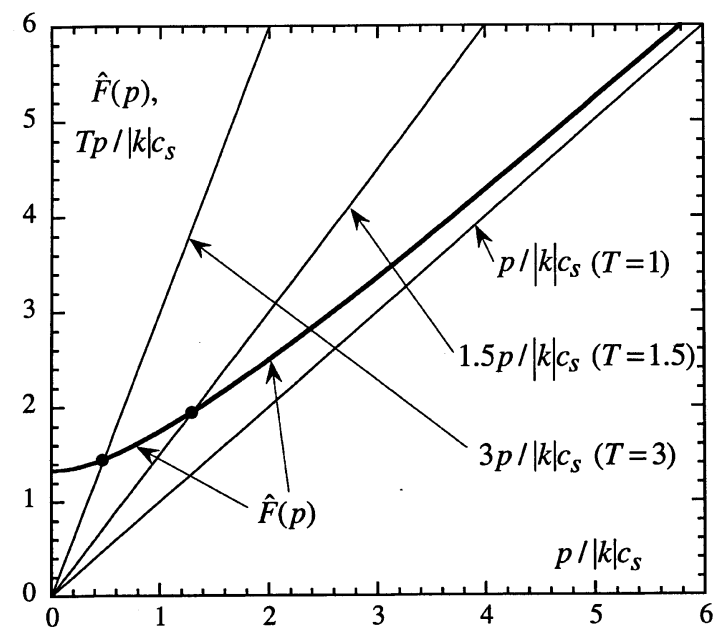

Fig. 5. For in-plane perturbations in the case of pure velocity dependent friction of weakening type: $T$ is a measure of the strength of velocity weakening and the solution of $\hat{F}(p)-T p /|k| c_{\mathrm{s}}=0$, with real $p$, is illustrated when $T>1$. The time dependence $\exp (p t)$ of response to an $\exp (\mathrm{i} k x)$ perturbation thus shows $p \propto|k| c_{\mathrm{s}}$, suggesting ill-posedness of the pure velocity-dependent model with $T>1$ for addressing the response to generic perturbations.

For non-zero $T$, that will have solutions only when $F(c)$ is imaginary, which means only when $c>c_{p}$, in which range the expression reduces to

$$
\frac{4 \sqrt{\left(c / c_{\mathrm{s}}\right)^{2}-1} \sqrt{\left(c / c_{p}\right)^{2}-1}+\left[2-\left(c / c_{\mathrm{s}}\right)^{2}\right]^{2}}{\left(c / c_{\mathrm{S}}\right)^{3} \sqrt{\left(c / c_{\mathrm{S}}\right)^{2}-1}}=T .
$$

As illustrated by the plot in Fig. 6 , done for $c_{p}=\sqrt{3} c_{\mathrm{s}}$, we find supersonic (relative to $c_{p}$ ) solutions in this case. That result is foreshadowed by Weertman's (1969) demonstration of similarly supersonic speeds of steady-state propagation of slip ruptures along surfaces with pure velocity-dependent friction of weakening type. The propagation speed $c$ becomes unbounded as $T \rightarrow 1^{-}$, but it reduces in magnitude (although always $>c_{p}$ ) as $T$ is reduced in size until at a special value, which we call $T_{\min }, c$ becomes equal to $c_{p}$. That lowest $T$ value is given by

$$
T_{\min }=\frac{\left[2-\left(c_{p} / c_{\mathrm{s}}\right)^{2}\right]^{2}}{\left(c_{p} / c_{\mathrm{s}}\right)^{3} \sqrt{\left(c_{p} / c_{\mathrm{s}}\right)^{2}-1}}
$$

which is $T_{\min }=1 /(3 \sqrt{6})=0.136$ for the case plotted in Fig. 6 . We have not determined the nature of the roots for $0<T<T_{\min }$. However, for $T=0$, the solution is the Rayleigh speed, $c=c_{\mathrm{R}}$ since $F\left(c_{\mathrm{R}}\right)=0$; that, of course, means neutrally stable (if not quasi-static) response to perturbation. Then, for small positive $T$, we can perturb about 


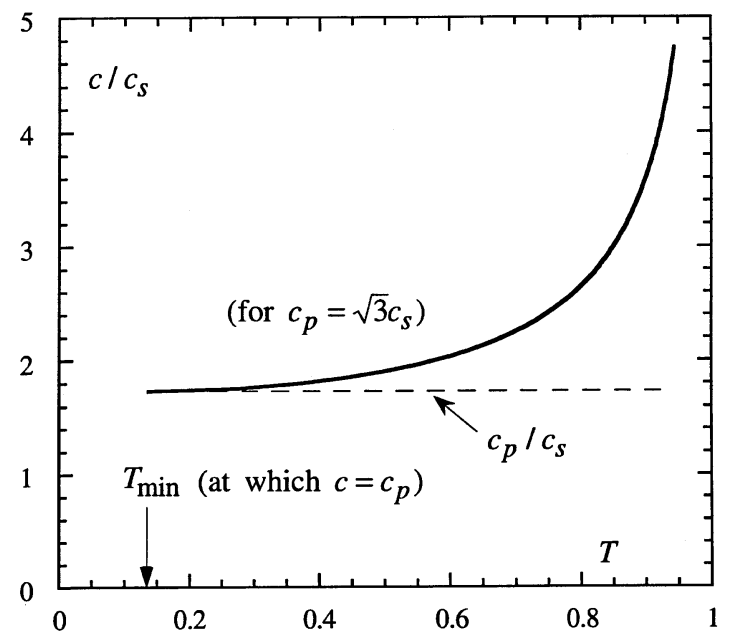

Fig. 6. Also for in-plane perturbations in the case of pure velocity-dependent friction of weakening type: $T$ is a measure of the strength of velocity weakening. When $T<1$ (but $T>T_{\min }$, see text) solutions of $\hat{F}(p)-T p /|k| c_{\mathrm{s}}=0$ have the form $p= \pm \mathrm{i}|k| c$, indicating a response to perturbation which propagates at speeds $\pm c$ along the sliding interface. However, in this range of $T$, the propagation speeds are supersonic, $c>c_{p}$.

that solution by Taylor expansion of $F(c)$. Thus, to linear order in $T$,

$$
F^{\prime}\left(c_{\mathrm{R}}\right)\left(\frac{p}{\mathrm{i}|k|}-c_{\mathrm{R}}\right)-\mathrm{i} T|k| c_{\mathrm{R}} / c_{\mathrm{s}}=0
$$

which shows that $p=\mathrm{i}|k| c_{\mathrm{R}}-T|k| c_{\mathrm{R}} /\left[c_{\mathrm{S}} F^{\prime}\left(c_{\mathrm{R}}\right)\right]$. Since $F^{\prime}\left(c_{\mathrm{R}}\right)<0$, this shows that $\operatorname{Re}(p)>0$, and further that $\operatorname{Re}(p) \propto|k|$, showing not only unstable modal response but also the feature that denotes ill-posedness in response to a generic perturbation.

\section{Quasistatic analysis of stability of slow frictional sliding between elastically dissimilar solids}

As explained towards the end of Section 1, frictional sliding with the positive direct effect should behave quasi-statically at sufficiently slow sliding rates $V_{0}$, i.e., when $V_{0} \ll 2 a \sigma_{0} c_{\mathrm{s}} / \mu$. Here, we analyze the stability of sliding in that quasi-static regime for the dissimilar materials case.

We denote by subscript " 1 " the material in $y>0$ and by " 2 " that in $y<0$. For sliding under quasi-static conditions between such elastically dissimilar materials, expressions for stress perturbations under in-plane slip perturbations can be taken from Comninou (1977a,b) and Comninou and Schmeuser (1979) (remembering that our $\tau=\sigma_{y x}$ and $\left.\sigma=-\sigma_{y y}\right)$ as

$$
\tau(x, t)-\tau_{0}=-\frac{M}{2 \pi} \int_{-\infty}^{+\infty} \frac{\partial \hat{\delta}(\xi, t) / \partial \xi}{x-\xi} \mathrm{d} \xi, \quad \sigma(x, t)-\sigma_{0}=\frac{M \beta}{2} \frac{\partial \hat{\delta}(x, t)}{\partial x},
$$


where $\hat{\delta}(x, t)=\hat{u}_{x}\left(x, 0^{+}, t\right)-\hat{u}_{x}\left(x, 0^{-}, t\right)$ is the slip perturbation. The modulus $M$ is twice a modulus $C$ introduced by Comninou and Schmeuser (1979) and $\beta$ is a Dundurs parameter. These are defined by

$$
\begin{aligned}
& M=\frac{8 \mu_{1} \mu_{2} /\left(1-\beta^{2}\right)}{\mu_{2}\left(\kappa_{1}+1\right)+\mu_{1}\left(\kappa_{2}+1\right)}=\frac{2 \mu_{1} \mu_{2} /\left(1-\beta^{2}\right)}{\mu_{2}\left(1-v_{1}\right)+\mu_{1}\left(1-v_{2}\right)}, \\
& \beta=\frac{\mu_{2}\left(\kappa_{1}-1\right)+\mu_{1}\left(\kappa_{2}-1\right)}{\mu_{2}\left(\kappa_{1}+1\right)+\mu_{1}\left(\kappa_{2}+1\right)}=\frac{\mu_{2}\left(1-2 v_{1}\right)-\mu_{1}\left(1-2 v_{2}\right)}{2\left[\mu_{2}\left(1-v_{1}\right)+\mu_{1}\left(1-v_{2}\right)\right]},
\end{aligned}
$$

where the rightmost versions correspond to plane strain $(\kappa=3-4 v)$ as treated here; the formulae are also valid for plane stress with $\kappa=(3-v) /(1+v)$. Our use of " 1 " and " 2 " is reversed relative to Comninou and Schmeuser (1979) but we use the expression as they wrote it for $\beta$, so that for a given material pair our $\beta$ is the negative of theirs, and has the property that when the more rigid material is the one occupying $y<0$, then $\beta>0$ (at least if the Poisson ratios of the two materials are not too dissimilar). Note that $M$ reduces to $\mu /(1-v)$ and $\beta=0$ in the case of identical materials treated in Sections 3.3 and 3.4. Suga et al. (1988) and Hutchinson and Suo (1992) give numerical values of $\beta$ for a wide range of material pairs. Typically, $|\beta|$ is of the order 0.1 or less although, for very dissimilar materials, e.g., with $\mu_{1} / \mu_{2} \rightarrow 0, \beta=\left(1-2 v_{1}\right) / 2\left(1-v_{1}\right)$ and can be as large as 0.5 if $v_{1}$ is near 0 .

Thus if we seek solutions in the form $\hat{\delta}(x, t)=A \exp (\mathrm{i} k x+p t)$, then the velocity perturbation is $V(x, t)-V_{0}=p A \exp (\mathrm{i} k x+p t)$ like in the previous cases and the stress perturbations are

$$
\begin{aligned}
& \tau(x, t)-\tau_{0}=-|k|(M / 2) A \exp (\mathrm{i} k x+p t), \\
& \sigma(x, t)-\sigma_{0}=\mathrm{i} k(M \beta / 2) A \exp (\mathrm{i} k x+p t) .
\end{aligned}
$$

Inserting these into the linearization of the constitutive relation, in its general form allowing for variations of normal stress, we obtain that, for a given real $k$, the time dependence $p$ must satisfy

$$
\frac{a \sigma_{0}}{V_{0}} p^{2}+\left[\frac{(a-b) \sigma_{0}}{L}+\frac{M|k|}{2}+\frac{\mathrm{i} k M \beta(f-\alpha)}{2}\right] p+\frac{V_{0}}{L}\left[\frac{M|k|}{2}+\frac{\mathrm{i} k M \beta f}{2}\right]=0,
$$

where we assume that $a>0$ and $L>0$. We now introduce the non-dimensional quantities

$$
n=M|k| L /\left(2 \sigma_{0}\right), \quad \varepsilon=\beta k /|k|, \quad \zeta=p L / V_{0}
$$

so that the expression reduces to

$$
a \zeta^{2}+\{n[1+\mathrm{i} \varepsilon(f-\alpha)]-(b-a)\} \zeta+n(1+\mathrm{i} \varepsilon f)=0 .
$$

In the special case of identical materials, $\beta=\varepsilon=0$. Then one sees by inspection that, if $a>b$ (steady-state velocity strengthening), there are no roots with $\operatorname{Re}(p)>0$ (i.e., with $\operatorname{Re}(\zeta)>0)$ and sliding is always stable. However, if $a<b$ (velocity weakening), there will be no roots with $\operatorname{Re}(p)>0$ if $n$ is large enough, but such root comes into 
existence when $n<n_{\mathrm{cr}}=b-a$. At $n=n_{\mathrm{cr}}$ the root is $\zeta= \pm \mathrm{i} \sqrt{(b-a) / a}$. Those reproduce the quasi-static results of the last section for in-plane perturbations.

Our aim now is to see how those conclusions change when the materials are dissimilar so that $\beta \neq 0$. We consider the nature of the roots $\zeta_{1}, \zeta_{2}$. If one is complex, the other need not be its complex conjugate. It is easy to show that if $n$ is sufficiently large, then both roots $\zeta_{1}, \zeta_{2}$ have negative real part. On the other hand, $\zeta=0$ can never be a root if $n>0$. Thus, as we consider progressively smaller wavenumbers (i.e., as we reduce $n$ from very large values), if one of $\zeta_{1}, \zeta_{2}$ develops a positive real part it must do so by crossing the imaginary $\zeta$ axis, which is a Hopf bifurcation. To establish that condition, we write $\zeta=\mathrm{i} \rho$, where $\rho$ is real, and seek to find the values, if any, of $n$ for a solution of that type to occur. With $\zeta=\mathrm{i} \rho$, the above quadratic equation in $\zeta$ becomes

$$
\left[-a \rho^{2}-n \varepsilon(f-\alpha) \rho+n\right]+\mathrm{i}[(n-a+b) \rho+\varepsilon f n]=0 .
$$

Setting the real part to 0 , one solves for

$$
\rho=\left[-n \varepsilon(f-\alpha) \pm \sqrt{n^{2} \varepsilon^{2}(f-\alpha)^{2}+4 a n}\right] / 2 a
$$

and, inserting that into the imaginary part, which must also be set to 0 , one finally obtains two tentative solutions for $n$. There are two such tentative solutions because there are two roots, which cross the imaginary $\zeta$-axis at different values of $n$, and the roots are tentative because, to be physically acceptable, they must provide a positive real number for $n$.

Calling the largest of the purported roots as $n_{\mathrm{cr}}$, since we will lose stability at the first axis crossing as we reduce $n$ from very large values, one obtains

$$
\begin{aligned}
{\left[1+\beta^{2}(f-\alpha) f\right] n_{\mathrm{cr}}=} & (b-a)+\left(\beta^{2} f / 2\right)[f a+(f-\alpha)(b-a)] \\
& +|\beta| f \sqrt{a(b-a)+\left(\beta^{2} / 4\right)[f a+(f-\alpha)(b-a)]^{2}} .
\end{aligned}
$$

The second root for $n$ is given by the same expression, but with a minus sign before the square root term.

If the $n_{\mathrm{cr}}$ so expressed is real and positive, then the bifurcation occurs at the critical value $k_{\mathrm{cr}}$ of $|k|$, and that critical wavenumber is

$$
k_{\mathrm{cr}} \equiv \frac{2 \pi}{\lambda_{\mathrm{cr}}}=\frac{2 n_{\mathrm{cr}} \sigma_{0}}{M L}
$$

Further, one can back substitute to find the value of $\rho$, and hence $p$, at the bifurcation. This is

$$
\begin{aligned}
p= & -i \operatorname{sgn}(k \beta) \frac{V_{0}}{L}\left[\sqrt{\frac{b-a}{a}+\frac{\beta^{2}}{4}\left(f+(f-\alpha) \frac{b-a}{a}\right)^{2}}\right. \\
& \left.+\frac{|\beta|}{2}\left|f+(f-\alpha) \frac{b-a}{a}\right|\right]
\end{aligned}
$$

which indicates that for dissimilar materials, in the sense $\beta \neq 0$ as now assumed, there is a unique direction of propagation of neutrally stable slip at the bifurcation point. 
That is, for $k=k_{\mathrm{cr}}$ and $k=-k_{\mathrm{cr}}$, the responses to perturbations take the form

$$
V(x, t)-V_{0}=A \exp \left[ \pm \mathrm{i} k_{\mathrm{cr}}(x-c t)\right],
$$

where $c$ (which is of magnitude $M V_{0} /\left[2 \sqrt{a(b-a)} \sigma_{0}\right]$ to lowest order in $\beta$ ) has the same sign as does $\beta$. Thus, if the two materials have comparable $v$, but the one in $y>0$ is less stiff, then $\beta>0$ and $c>0$. The direction of propagation is the same as the direction of sliding of the more compliant material relative to the more rigid one. This echoes results from the dynamic analysis of sliding in dissimilar materials, although as mentioned, those have been done so far only in the cases of classical Coulomb friction, and of a particular regularization of it (Ranjith and Rice, 2001; Cochard and Rice, 2000) that corresponds to $\alpha=f$ and $a=b=0$ here.

Let us now analyze the conditions for which the solution just given makes the expression for $n_{\text {cr }}$ real and positive, so that there is instability of sliding in response to perturbations of sufficiently low wavenumbers. Clearly, that condition is met whenever $b>a$ (i.e., when there is velocity weakening). However, one might suspect that if $a>b$ (velocity strengthening), then there may be a critical value of $a-b$ such that any stronger velocity strengthening will assure that sliding is stable to perturbations of all wavelengths, which is to say that no real positive solution for $n_{\text {cr }}$ exists. As we consider increase of $a-b$ from 0 , it is easy to see that the expression under the square root, in the equation above for $n_{\mathrm{cr}}$, passes from positive to negative before the remaining part of the right side of that equation does. Vanishing of that square root term thus gives the critical velocity strengthening as

$$
(a-b)_{\text {crit }}=\frac{\beta^{2} f^{2} a / 2}{\left[1+\beta^{2} f(f-\alpha) / 2\right]+\sqrt{1+\beta^{2} f(f-\alpha)}} .
$$

Since $\beta^{2}$ is generally much less than unity (Suga et al., 1988; Hutchinson and Suo, 1992), and $f$ is seldom as large as unity, in essentially all practical cases this will reduce to

$$
(a-b)_{\text {crit }}=\beta^{2} f^{2} a / 4
$$

as the minimum value of velocity strengthening necessary to counteract the destabilizing effects of slip-to-normal-stress coupling and assure that the sliding dissimilar material interface is stable to perturbations of all wavelengths. We leave it to future work to understand how this result might change when velocities are not so small as to satisfy $V_{0} \ll 2 a \sigma_{0} c_{\mathrm{s}} / \mu$, the quasi-static condition given earlier and discussed further in the next section.

\section{Perspectives on the quasi-static range from elastodynamic equations for dissimilar materials with rate and state dependent friction}

Here, we study dynamic stability to linearized perturbations from a state of steady sliding along an interface between dissimilar materials and confirm the result of our quasi-static analysis (Section 4) that the perturbations with high wavenumbers are always stable for sufficiently low sliding velocities. Hence, we establish that a rate 
and state dependent friction law with a positive direct effect, $a>0$, gives rise to a well-posed stability problem in the limit of sufficiently low sliding velocity $V_{0}$.

As before the unperturbed state is one of spatially uniform steady-state sliding at velocity $V_{0}$, a situation for which the shear and normal stresses are uniform at $\tau_{\mathrm{o}}$ and $\sigma_{0}$. Suppose that at times $t>0$, a perturbation in the shear stress at the interface of the form

$$
\Delta \tau=Q \exp (\mathrm{i} k x+p t)
$$

is imposed. We define this perturbation such that, if the interfacial points were constrained to continue to slide at the rate $V_{0}$ while a shear perturbation loading was applied, then the extra shear traction $Q \exp (\mathrm{i} k x+p t)$ would have to be borne at the interface, but no extra normal traction. In the absence of any such constraint, the linearized response in shear and normal stresses and in slip velocity will then take the form

$$
\tau(x, t)-\tau_{0}=T_{1} \exp (\mathrm{i} k x+p t), \quad \sigma(x, t)-\sigma_{0}=-T_{2} \exp (\mathrm{i} k x+p t)
$$

and

$$
V(x, t)-V_{0}=p D_{1} \exp (\mathrm{i} k x+p t) .
$$

Following Ranjith and Rice (2001), it can be shown the equations of elastodynamics will be satisfied in the two half-spaces if the relationship between these perturbations is

$$
T_{1}=M_{11} D_{1}+Q, \quad T_{2}=M_{21} D_{1} .
$$

The explicit form of the transfer functions $M_{i j}$ involves rather complicated expressions, that can be developed from Geubelle and Rice (1995) and are given in Ranjith and Rice (2001). Neglecting the $Q$ term, these are $\{T\}=[M]\{D\}$ where $\{T\}=\left(T_{1}, T_{2}\right)^{\prime}$ and $\{D\}=\left(D_{1}, D_{2}\right)^{\prime}$ where $D_{2}$, the interface opening displacement, is set to zero. Ranjith and Rice began with equations in the form $\{D\}=[K]\{T\}$, natural because tractions are continuous across the interface, and obtained $[M]$ as $[K]^{-1}$, so that

$$
M_{11}=K_{22} / \Delta, \quad M_{21}=-K_{21} / \Delta=K_{12} / \Delta, \quad \Delta=\operatorname{det}[K]=K_{11} K_{22}+K_{12}^{2} .
$$

For our purposes here, we note that the $[M]$ have the form

$$
M_{i j}=\mu|k| Y_{i j}(P),
$$

where $\mu$ is a representative shear modulus of the bi-material pair and $P=p /|k| c_{\mathrm{s}}$ with $c_{\mathrm{s}}$ being a representative shear wave speed. The functions $Y_{i j}(P)$ are dimensionless, but depend on $k /|k|$ and, of course, on ratios of density and moduli of the bimaterial pair.

When we require that these perturbations also satisfy the linearized friction law at the interface, there results an equation determining the slip perturbation in the form

$$
D_{1}=(P+\hat{v}) Q /[\mu|k| P g(P)],
$$

where $\hat{v}=V_{0} /\left(c_{\mathrm{s}}|k| L\right)$ and

$$
\begin{aligned}
g(P)= & \left(\sigma_{0} c_{\mathrm{s}} / \mu V_{0}\right)[a P+(a-b) \hat{v}]-\left[Y_{11}(P)+(f-\alpha) Y_{21}(P)\right] \\
& -\hat{v}\left[Y_{11}(P)+f Y_{21}(P)\right] / P .
\end{aligned}
$$


For the stability problem to be well-posed, we require that there be no zero of $g(P)$ with positive real part in the short wavelength limit, $|k| \rightarrow \infty$. We show that is indeed the case at sufficiently low sliding velocities (the quasi-static limit) as long as $a>0$. In the limit $|k| \rightarrow \infty$,

$$
g(P)=\left(a \sigma_{0} c_{\mathrm{s}} / \mu V_{0}\right) P-Y_{11}(P)-(f-\alpha) Y_{21}(P) .
$$

First, consider the case where $a=0$. Then $g(P)$ becomes $-Y_{11}(P)-(f-\alpha) Y_{21}(P)$ and is precisely of the form derived by Ranjith and Rice (2001) (see their Eq. (28)) that governs stability to perturbations at a sliding dissimilar material interface with a Coulomb friction law. It follows from their analysis that $g(P)$ has a zero with positive real part for a wide range of material combinations and values of $f$ and $\alpha$, giving rise to ill-posedness. In particular, the stability results depend on the existence of an interfacial wave, called the generalized Rayleigh wave (Weertman, 1963; Achenbach and Epstein, 1967; Gol'dshtein, 1967), in frictionless sliding of the two half-spaces. If the material parameters are such that the generalized Rayleigh wave exists in frictionless sliding, $g(P)$ has a zero in the right-half $P$-plane for any $f$ and $\alpha$ as long as $(f-\alpha) \neq 0$. If the generalized Rayleigh wave does not exist for the bimaterial pair, a right-half plane zero exists if $|f-\alpha|$ is greater than a critical value, dependent on the bimaterial pair. The stability problem is therefore quite often ill-posed when $a=0$.

Next, we let $a \neq 0$ and rewrite $g(P)$ in the form

$$
g(P)=P / \varepsilon+Y(P)
$$

where $\varepsilon=\mu V_{0} / a \sigma_{0} c_{\mathrm{s}}(>0)$ and $Y(P)=-Y_{11}(P)-(f-\alpha) Y_{21}(P)$. In the following, we assume the sliding velocity $V_{0}$ is sufficiently low that $\varepsilon \ll 1$. Then, the roots of $g(P)$ must lie either close to the origin or close to a singularity of $Y(P)$. In the following, we show that all such possible roots have negative real parts.

It is easily seen that the root close to the origin will lie at $P=-\varepsilon Y(0)$. Since $Y_{21}(0)$ is purely imaginary, the real part of the root is $\varepsilon Y_{11}(0)$. Observing that $Y_{11}(0)$ relates the static slip distribution in a single $\exp (\mathrm{i} k x)$ mode to the shear stress in that mode at the interface, the requirement of a positive definite strain energy density function necessitates $Y_{11}(0)<0$. (These features of $Y_{21}(0)$ and $Y_{11}(0)$ may also be directly verified from the Comninou and Schmeuser (1979) static solution of Section 4 , showing that $M_{11}=-|k| M / 2$ and $M_{21}=-\mathrm{i} k M \beta / 2$ at $P=0$, where $M>0$.) Therefore, since $\varepsilon>0$, the root close to the origin always lies in the left-half $P$-plane.

Let us consider now the roots close to singularities of $Y(P)$. From the expressions in Ranjith and Rice (2001) for $Y_{11}(P)$ and $Y_{21}(P)$, it is seen that $Y(P)$ is $O(P)$ as $P \rightarrow \infty$ and hence singular there (indeed, this is the singularity that gives rise to the radiation damping effect). Inspection of the form of $g(P)$ immediately informs that this singularity is not strong enough for a root to exist close to $P=\infty$. The only other condition when a singularity of $Y(P)$ can exist, if at all, would be for purely imaginary $P$ (otherwise energy conservation would be violated for an interface with continuous 
displacements, hence no source of dissipation). That occurs when a Stonely wave exists for the bimaterial pair. That is a wave for which displacements are fully continuous, $\{D\}=\{0\}$, so that its condition is that $[K]$ be singular, i.e., that $\Delta=0$. When the Stonely wave exists, all the $Y_{i j}(P)$ are singular at $p= \pm|k| \mathrm{i} c_{\mathrm{St}}$ (or $P= \pm \mathrm{i} c_{\mathrm{St}} / c_{\mathrm{S}}$ ), where $c_{\mathrm{St}}$ is the speed of propagation of the Stonely wave.

We show in the remainder of this section that, in such cases, the root close to $P= \pm \mathrm{i} c_{\mathrm{St}} / c_{\mathrm{S}}$ lies in the left-half $P$-plane if $\varepsilon>0$. Therefore, if the direct velocity effect component, $a$, of the friction law is positive and the sliding velocity is low enough that $\mu V_{0} / a \sigma_{0} c_{\mathrm{s}} \ll 1$, the problem of stability to perturbations from steady sliding at a dissimilar material interface is always well-posed.

For material pairs for which $c_{\mathrm{St}}$ exists, one has the singular structure

$$
Y_{11}(P) \sim \frac{\mathrm{i} A}{\mathrm{i}\left(c_{\mathrm{St}} / c_{\mathrm{s}}\right)-P}, \quad Y_{21}(P) \sim \frac{B}{\mathrm{i}\left(c_{\mathrm{St}} / c_{\mathrm{s}}\right)-P}
$$

near $P=\mathrm{i}\left(c_{\mathrm{St}} / c_{\mathrm{S}}\right)$, where $A$ and $B$ are real (from expressions for $[K]$ and $[M]$ in Ranjith and Rice, 2001). Hence, when $\varepsilon \ll 1$, there is a solution of $g(P)=0$ near the pole given to leading order by

$$
\frac{\mathrm{i}\left(c_{\mathrm{St}} / c_{\mathrm{s}}\right)}{\varepsilon}-\frac{\mathrm{i} A}{\mathrm{i}\left(c_{\mathrm{St}} / c_{\mathrm{S}}\right)-P}-\frac{(f-\alpha) B}{\mathrm{i}\left(c_{\mathrm{St}} / c_{\mathrm{S}}\right)-P} \sim 0
$$

giving $P \sim \mathrm{i}\left(c_{\mathrm{St}} / c_{\mathrm{S}}\right)-\varepsilon\left(c_{\mathrm{S}} / c_{\mathrm{St}}\right)[A-\mathrm{i}(f-\alpha) B]$. Hence, provided that we can show that $A>0$, the root lies in the left-half $P$-plane.

We now note, following Weertman (1963), Achenbach and Epstein (1967), and Gol'dshtein (1967), that relevant wave speeds for bimaterial problems are the generalized Rayleigh speed $c_{\mathrm{GR}}$, for frictionless slip without opening, a companion wave speed $c_{\mathrm{Op}}$ for unimpeded opening without slip, and $c_{\mathrm{St}}$ for the case of there being neither slip nor opening. These speeds are taken as positive here and provide the roots $p= \pm \mathrm{i}|k| c_{\mathrm{GR}}, \pm \mathrm{i}|k| c_{\mathrm{Op}}$ and $\pm \mathrm{i}|k| c_{\mathrm{St}}$ to the respective equations, $K_{22}(p)=0$, $K_{11}(p)=0$ and $\Delta(p)=0$, when those equations have roots. Without loss of generality, we order the materials of Fig. 1 so that $c_{\mathrm{R} 1}<c_{\mathrm{R} 2}$. Then all these speeds $c$ satisfy $c_{\mathrm{R} 1}<c<\min \left(c_{\mathrm{R} 2}, c_{\mathrm{s} 1}\right)$. We note that along the imaginary axis $p=\mathrm{i}|k| c$, where $c$ is real and $0<c<\min \left(c_{\mathrm{s} 1}, c_{\mathrm{s} 2}\right), K_{11}$ and $K_{22}$ are real, whereas $K_{12}$ is pure imaginary, which means that $\Delta=K_{11} K_{22}-\left|K_{12}\right|^{2}$ there. We also prove an ordering of the speeds as follows: Standing vibrations may be composed by superposing solutions of the type $\exp [\mathrm{i} k(x-c t)]$ and $\exp [\mathrm{i} k(x+c t)]$, and these have frequency $|k| c$. By Rayleigh's quotient, since the displacement field of the Stonely mode is kinematically admissible for the other two modes, it is of higher frequency and hence $\max \left(c_{\mathrm{GR}}, c_{\mathrm{Op}}\right) \leqslant c_{\mathrm{St}}$. Study of the expressions for the $[K]$ in Ranjith and Rice, and the results of Weertman, Achenbach and Epstein, and Gol'dshtein mentioned above, then shows the following: (i) $c_{\mathrm{Op}}$ always exists, no matter what the bimaterial pair. (ii) $c_{\mathrm{GR}}$ exists only for bimaterial pairs that are not too dissimilar; it always exists if $c_{\mathrm{R} 2}<c_{\mathrm{S} 1}$ but goes out of existence if $c_{\mathrm{R} 2}$ is too much larger than $c_{\mathrm{s} 1}$. (iii) $c_{\mathrm{St}}$ may exist only for bimaterial pairs for which $c_{\mathrm{GR}}$ exists. That follows because, if $c_{\mathrm{GR}}$ does not exist, which is the case when $c_{\mathrm{R} 2}$ is sufficiently greater than $c_{\mathrm{s} 1}$, then examination of the expressions for the $[K]$ shows 
that $K_{11}<0$ and $K_{22}>0$ in the range $c_{\mathrm{Op}}<c<c_{\mathrm{s} 1}$ in which $c_{\mathrm{St}}$ would have to lie, if it exists. But $K_{11}<0$ and $K_{22}>0$ imply that $\Delta<0$, so that $\Delta=0$ has no solution in that range, and hence $c_{\mathrm{St}}$ cannot exist.

Now, assuming that the bimaterial pair is such that $c_{S t}$ exists, which we have just seen to require that $c_{\mathrm{GR}}$ does also, the Stonely pole factor $A$ will be positive if $M_{11}=$ $K_{22} / \Delta>0$ for $\max \left(c_{\mathrm{GR}}, c_{\mathrm{Op}}\right)<c<c_{\mathrm{St}}$. From the expressions for $K_{11}$ and $K_{22}$ in Ranjith and Rice (2001), and recalling that $c_{\mathrm{Op}}$ and $c_{\mathrm{GR}}$ are the respective zeros of $K_{11}$ and $K_{22}$, we have that $K_{11}$ and $K_{22}$ are of opposite sign for $\min \left(c_{\mathrm{GR}}, c_{\mathrm{Op}}\right)<c<\max \left(c_{\mathrm{GR}}, c_{\mathrm{Op}}\right)$ and hence that $\Delta<0$ for $c$ in that range. However, since $c_{\mathrm{St}}$ is the root of $\Delta=0$, that implies that $\Delta<0$ for $\max \left(c_{\mathrm{GR}}, c_{\mathrm{Op}}\right)<c<c_{\mathrm{St}}$. In that same range of $c$, which is the range where $c$ is greater than the roots of $K_{11}$ and $K_{22}$, the expressions of Ranjith and Rice show that both $K_{11}$ and $K_{22}$ are negative. Thus $K_{22} / \Delta>0$ for $\max \left(c_{\mathrm{GR}}, c_{\mathrm{Op}}\right)<c$ $<c_{\mathrm{St}}$, which proves that $A>0$ and hence that the root near the Stonely pole lies in the domain $\operatorname{Re}(P)<0$.

That shows, finally, that the problem of stability to perturbations from steady sliding at a dissimilar material interface is always well-posed in the high wavenumber limit, at sliding velocity that is low enough that $\mu V_{0} / a \sigma_{0} c_{\mathrm{s}} \ll 1$. This condition can, of course, be met only if the direct effect, $a$, of rate and state friction is present in the friction model.

\section{Conclusions}

We have established conditions for the stability of steady frictional sliding between two elastically deformable half-spaces, assuming physically based friction laws of the rate and state dependent type. Of particular importance to controlling the nature of the response to perturbation, and how it may differ qualitatively from response predicted with classical friction laws, is the positive "direct effect" of slip rate change in rate and state laws. That effect is measured by the parameter $a$, where $a>0$. Typically, $a$ for rocks is of order 0.01 . Rate and state laws were used in their simplest form for the stability investigations here, by adopting a single evolving state variable to characterize strength changes due to changes in maturity of the population of contacting asperities (the basis for velocity weakening). We have also reviewed, and slightly generalized, procedures for writing rate and state friction laws for conditions of variable normal stress.

The direct effect is thought to be due to thermally activated slip at asperity contacts. We have estimated activation energies (1.7-1.8 eV) and volumes (a few atomic volumes) for the process from room-temperature data for quartzite and granite. That basis for $a$ explains why the shear stress response to an instantaneous change in slip rate $V$, at constant normal stress $\sigma$, has approximately the form $\partial \tau / \partial V=a \sigma / V$, with $a>0$ and constant (at a given temperature), except at such extremely low slip rates that backward jumps can no longer be neglected in the thermal activation model. Comparing $\partial \tau / \partial V$ so expressed to a characteristic dynamic parameter $\mu / 2 c_{\mathrm{s}}$ (the radiation damping factor for sliding), we see that $\partial \tau / \partial V$ can always be made to dominate the dynamic term if $V$ is small enough, that is, if $V \ll 2 a \sigma c_{\mathrm{s}} / \mu$. That provides a quantification of 
"slow" frictional sliding, at least relative to elastodynamic effects. Such a regime does not exist if the direct effect is neglected.

For sliding between identical elastic solids, with rate and state friction involving steady-state velocity weakening at their interface, we have shown the following: The response to perturbations of type $\exp (\mathrm{i} k x)$ is always stable when $|k|$ is sufficiently large. There is a critical value of $|k|$, called $k_{\mathrm{cr}}$ (expressed in terms of parameters of the friction law, elastic properties, $\sigma$ and, at higher slip rates, $V$ ), below which there is instability. The instability always occurs as a Hopf bifurcation so that the response to slip perturbations at the critical wave number is of the form $\exp \left[\mathrm{i} k_{\mathrm{cr}}(x \pm c t)\right]$. The phase velocity $c$ (taken non-negative) always satisfies $c<c_{\mathrm{s}}$ for anti-plane perturbations, and $c<c_{\mathrm{R}}$ for in-plane.

Those results relied on the property $a>0$. In contrast, if one neglects the actual rate and state features of friction, and assumes purely rate-dependent friction, of velocity-weakening type, then we show the following: There is instability for all $k$. The response $\exp (\mathrm{i} k x) \exp (p t)$ to perturbation, when a dimensionless measure (called $T$ ) of the magnitude of the velocity weakening satisfies $T>1$, has the form $p \propto|k|$, with positive coefficient of proportionality. That means that the problem of response to a generic perturbation is ill-posed. When there is a smaller magnitude of velocity weakening, so that $T<1$ (but, nevertheless $T>T_{\min }$, a small threshold, in the in-plane case), the response is of the form $\exp [\mathrm{i} k(x \pm c t)]$, but with the paradoxical feature that $c$ is supersonic. That is, $c>c_{\mathrm{s}}$ for anti-plane perturbations and $c>c_{p}$ for in-plane.

For sliding between elastically dissimilar materials, a full dynamic analysis of stability to perturbation, in the rate and state friction context, has not yet been worked out. An additional source of instability, other than possible velocity weakening of friction, is that spatially inhomogeneous slip causes an alteration of normal stress; reductions of normal stress can reduce frictional resistance and thus destabilize. When a classical friction law with constant fiction coefficient is used, that dissimilar material problem is known to often (depending on friction level and ratios of material properties) be ill-posed. We show that with use of rate and state laws, the response to perturbation at very short wave lengths (high $|k|$ ) is stable at sufficiently low slip rates, in the sense that the $\exp (\mathrm{i} k x) \exp (p t)$ response has $\operatorname{Re}(p)<0$, suggesting that the problem is then well-posed.

Assuming tentatively that with rate and state friction, a quasi-static range of response exists in that dissimilar material case, and will coincide with a full dynamic analysis at low enough slip rates, we have solved the quasi-static problem of response to exp $(\mathrm{i} k x)$ perturbations. This shows stability of response at large $|k|$, but the possibility of unstable response as $|k|$ is reduced in size. That instability can occur even if there is steady-state velocity strengthening. We establish the critical magnitude of the velocity strengthening so that no $k_{\mathrm{cr}}$ exists, i.e., so that sliding is stable to perturbations of all wavelengths.

\section{Acknowledgements}

This study was supported by grants from the Office of Naval Research, the US Geological Survey, and the NSF Southern California Earthquake Center. We are grateful 
to C. Marone, M. Nakatani and H. Perfettini for discussions on friction laws, and to R. Madariaga for discussions on purely velocity-dependent friction models of weakening type and supersonic propagation.

\section{References}

Achenbach, J.D., Epstein, H.I., 1967. Dynamic interaction of a layer and a half-space. ASCE J. Eng. Mech. Div. EM 5, 27-42.

Adams, G.G., 1995. Self-excited oscillations of two elastic half-spaces sliding with a constant coefficient of friction. J. Appl. Mech. 62, 867-872.

Andrews, D.J., Ben-Zion, Y., 1997. Wrinkle-like slip pulse on a fault between different materials. J. Geophys. Res. 102, 553-571.

Baumberger, T., 1997. Contact dynamics and friction at a solid-solid interface: material versus statistical aspects. Solid State Commun. 102, 175-185.

Baumberger, T., Berthoud, P., Caroli, C., 1999. Physical analysis of the state- and rate-dependent friction law. II. Dynamic friction Phys. Rev., B 60.

Ben-Zion, Y., Rice, J.R., 1997. Dynamic simulations of slip on a smooth fault in an elastic solid. J. Geophys. Res. 102, 17,771-17,784.

Ben-Zion, Y., 2001. Dynamic ruptures in recent models of earthquake faults. J. Mech. Phys. Solids 49, 2209-2244.

Boitnott, G.N., Biegel, R.L., Scholz, C.H., Yoshioka, N., Wang, W., 1992. Micromechanics of rock friction 2: Quantitative modeling of initial friction with contact theory. J. Geophys. Res. 97, 8965-8978.

Bréchet, Y., Estrin, Y., 1994. The effect of strain rate sensitivity on dynamic friction of metals. Scripta Met. Mater. 30, 1449-1454.

Bureau, L., Baumberger, T., Caroli, C., 2000. Shear response of a frictional interface to a normal load modulation. Phys. Rev. E 62, 6810-6820.

Chester, F.M., 1994. Effects of temperature on friction: constitutive equations and experiments with fault gouge. J. Geophys. Res. 99, 7247-7261.

Cochard, A., Rice, J.R., 2000. Fault rupture between dissimilar materials: Ill-posedness, regularization, and slip-pulse response. J. Geophys. Res. 105, 25,891-25,907.

Comninou, M., 1977a. A property of interface dislocations. Philos. Mag. 36, 1281-1283.

Comninou, M., 1977b. The interface crack. J. Appl. Mech. 44, 631-636.

Comninou, M., Schmeuser, D., 1979. The interface crack in a combined tension-compression and shear field. J. Appl. Mech. 49, 345-348.

Dieterich, J.H., 1979. Modeling of rock friction-1. Experimental results and constitutive equations. J. Geophys. Res. 84, 2161-2168.

Dieterich, J.H., 1981. Constitutive properties of faults with simulated gouge. In: Carter, N.L., Friedman, M., Logan, J.M., Stearns, D.W. (Eds.), Mechanical Behavior of Crustal Rocks., Geophysical Monograph Series, vol. 24. American Geophysical Union, Washington, DC, pp. 103-120.

Dieterich, J.H., Kilgore, B.D., 1994. Direct observation of frictional contacts: new insights for state dependent properties. Pure Appl. Geophys. 143, 283-302.

Dieterich, J.H., Kilgore, B.D., 1996. Imaging surface contacts: power law contact distributions and contact stresses in quartz, calcite, glass and acrylic plastic. Tectonophysics 256, 219-239.

Geubelle, P.H., Rice, J.R., 1995. A spectral method for three-dimensional elastodynamic fracture problems. J. Mech. Phys. Solids 43, 1791-1824.

Gol'dshtein, R.V., 1967. On surface waves in joined elastic media and their relation to crack propagation along the junction. PMM 31(3), 468-475. (English translation, Appl. Math. Mech. 31, 496-502, 1967).

Heslot, F., Baumberger, T., Perrin, B., Caroli, B., Caroli, C., 1994. Creep, stick-slip, and dry friction dynamics: experiments and a heuristic model. Phys. Rev. E 49, 4973-4988.

Hutchinson, J.W., Suo, Z., 1992. Mixed mode cracking in layered materials. In: Hutchinson, J.W., Wu, T.Y. (Eds.), Advances in Applied Mechanics, vol. 29. Academic Press, New York, pp. 63-191.

Knopoff, L., Landoni, J.A., 1998. Noncausality of fractures with linear strength weakening, unpublished manuscript. 
Lapusta, N., Rice, J.R., Ben-Zion, Y., Zheng, G., 2000. Elastodynamic analysis for slow tectonic loading with spontaneous rupture episodes on faults with rate- and state dependent friction. J. Geophys. Res. 105, $23,765-23,789$.

Linker, M.F., Dieterich, J.H., 1992. Effects of variable normal stress on rock friction: Observations and constitutive equations. J. Geophys. Res. 97, 4923-4940.

Marone, C., 1998. Laboratory-derived friction laws and their application to seismic faulting. Ann. Rev. Earth Planetary Sci. 26, 643-696.

Martins, J.A.C., Faria, L.O., Guimarães, J., 1992. Dynamic surface solutions in linear elasticity with frictional boundary conditions. In: Ibrahim, R.A., Soom, A. (Eds.), Friction-Induced Vibration, Chatter, Squeal and Chaos, DE-Vol. 49. ASME, New York, pp. 33-39.

Martins, J.A.C., Guimarães, J., Faria, L.O., 1995. Dynamic surface solutions in linear elasticity and viscoelasticity with frictional boundary conditions. J. Vib. Acous. Trans. ASME 117, 445-451.

Martins, J.A.C., Simões, F.M.F., 1995. On some sources of instability/ill-posedness in elasticity problems with Coulomb friction. In: Raous, M., Jean, M., Moreau, J.J. (Eds.), Contact Mechanics. Plenum Press, New York, pp. 95-106.

Nakatani, M., 2001. Physical clarification of rate and state dependent friction law: Thermally activated rheology of frictional sliding. J. Geophys. Res., in press.

Oden, J.T., Martins, J.A.C., 1985. Models and computational methods for dynamic friction phenomena. Comput. Meth. Appl. Mech. Eng. 52, 527-634.

Perfettini, H., Schmittbuhl, J., Rice, J.R., Cocco, M., 2001. Frictional response induced by time-dependent fluctuations of the normal loading. J. Geophys. Res., in press.

Perrin, G., Rice, J.R., Zheng, G., 1995. Self-healing slip pulse on a frictional surface. J. Mech. Phys. Solids 43, 1461-1495.

Persson, B.N.J., 1998. Sliding Friction: Physical Principles and Applications. Springer, Heidelberg.

Prakash, V., 1998. Frictional response of sliding interfaces subjected to time varying normal pressures. J. Tribol. Trans. ASME 120, 97-102.

Prakash, V., Clifton, R.J., 1992. Pressure-shear plate impact measurement of dynamic friction for high speed machining applications. Proceedings of the Seventh International Congress on Experimental Mechanics. Society of Experimental Mechanics, Bethel, Conn., pp. 556-564.

Ranjith, K., Rice, J.R., 2001. Slip dynamics at an interface between dissimilar materials. J. Mech. Phys. Solids 49, 341-361.

Renardy, M., 1992. Ill-posedness at the boundary for elastic solids sliding under Coulomb friction. J. Elasticity 27, 281-287.

Rice, J.R., 1983. Constitutive relations for fault slip and earthquake instabilities. Pure Appl. Geophys. 121, $443-475$.

Rice, J.R., Ruina, A.L., 1983. Stability of steady frictional slipping. J. Appl. Mech. 50, 343-349.

Rice, J.R., Ben-Zion, Y., 1996. Slip complexity in earthquake fault models. Proc. Nat. Acad. Sci. USA 93, 3811-3818.

Richardson, E., Marone, C., 1999. Effects of normal force vibrations on frictional healing. J. Geophys. Res. $104,28,859-28,878$.

Ruina, A.L., 1980. Friction laws and instabilities: a quasistatic analysis of some dry frictional behavior. Ph.D. Thesis, Division of Engineering, Brown University, Providence, Rhode Island.

Ruina, A.L., 1983. Slip instability and state variable friction laws. J. Geophys. Res. 88, 10,359-10,370.

Segall, P., Rice, J.R., 1995. Dilatancy, compaction, and slip instability of a fluid infiltrated fault. J. Geophys. Res. 100, 22,155-22,171.

Simões, F.M.F., Martins, J.A.C., 1998. Instability and ill-posedness in some friction problems. Int. J. Eng. Sci. 36, 1265-1293.

Sleep, N.H., 1995. Ductile creep, compaction, and rate and state dependent fiction within major fault zones J. Geophys. Res. 100, 13,065-13,080.

Sleep, N.H., 1997. Application of a unified rate and state friction theory to the mechanics of fault zones with strain localization. J. Geophys. Res. 102, 2875-2895.

Stesky, R.M., 1977. Mechanisms of high temperature frictional sliding in Westely granite. Can. J. Earth Sci. $15,361-375$. 
Suga, T., Elssner, E., Schmauder, S., 1988. Composite parameters and mechanical compatibility of material joints. J. Composite Mater. 22, 917-934.

Tullis, T.E., Weeks, J.D., 1986. Constitutive behavior and stability of fictional sliding in granite. Pure Appl. Geophys. 124, 383-414.

Weertman, J., 1963. Dislocations moving uniformly on the interface between isotropic media of different elastic properties. J. Mech. Phys. Solids 11, 197-204.

Weertman, J., 1969. Dislocation motion on an interface with friction that is dependent on sliding velocity. J. Geophys. Res. 74, 6617-6622.

Weertman, J., 1980. Unstable slippage across a fault that separates elastic media of different elastic constants. J. Geophys. Res. 85, 1455-1461. 